\title{
Development and Commercialization of Alternative Carbon Fiber Precursors and Conversion Technologies
}

April 2014

Prepared by

Robert E. Norris

Approved for public release; distribution is unlimited.

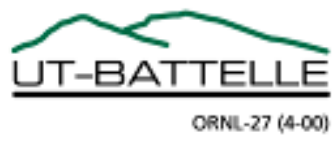




\title{
DOCUMENT AVAILABILITY
}

Reports produced after January 1, 1996, are generally available free via the U.S. Department of Energy (DOE) Information Bridge.

Web site http://www.osti.gov/bridge

Reports produced before January 1, 1996, may be purchased by members of the public from the following source.

\author{
National Technical Information Service \\ 5285 Port Royal Road \\ Springfield, VA 22161 \\ Telephone 703-605-6000 (1-800-553-6847) \\ TDD 703-487-4639 \\ Fax 703-605-6900 \\ E-mail info@ntis.gov \\ Web site http://www.ntis.gov/support/ordernowabout.htm
}

Reports are available to DOE employees, DOE contractors, Energy Technology Data Exchange (ETDE) representatives, and International Nuclear Information System (INIS) representatives from the following source.

Office of Scientific and Technical Information

P.O. Box 62

Oak Ridge, TN 37831

Telephone 865-576-8401

Fax 865-576-5728

E-mail reports@osti.gov

Web site http://www.osti.gov/contact.html

This report was prepared as an account of work sponsored by an agency of the United States Government. Neither the United States Government nor any agency thereof, nor any of their employees, makes any warranty, express or implied, or assumes any legal liability or responsibility for the accuracy, completeness, or usefulness of any information, apparatus, product, or process disclosed, or represents that its use would not infringe privately owned rights. Reference herein to any specific commercial product, process, or service by trade name, trademark, manufacturer, or otherwise, does not necessarily constitute or imply its endorsement, recommendation, or favoring by the United States Government or any agency thereof. The views and opinions of authors expressed herein do not necessarily state or reflect those of the United States Government or any agency thereof. 
Advanced Manufacturing Office

\title{
Development and Commercialization of Alternative Carbon Fiber Precursors and Conversion Technologies
}

\author{
Authors \\ Robert E. Norris, Michael Kaufman, Amit Naskar, Felix Paulauskas, Ken Yarborough \\ Chris Derstine, The Dow Chemical Company
}

April 2014

Prepared by

OAK RIDGE NATIONAL LABORATORY

Oak Ridge, Tennessee 37831-6283

managed by

UT-BATTELLE, LLC

for the

U.S. DEPARTMENT OF ENERGY

under contract DE-AC05-00OR22725 



\section{CONTENTS}

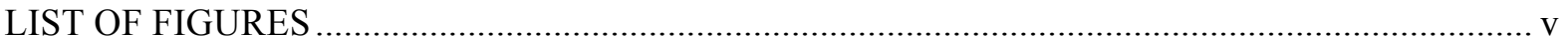

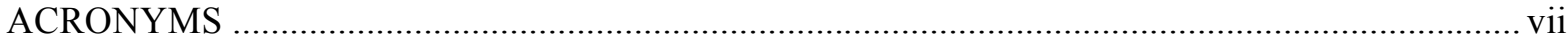

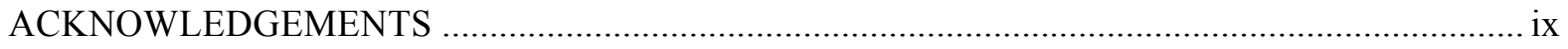

EXECUTIVE SUMMARY .........................................................................................

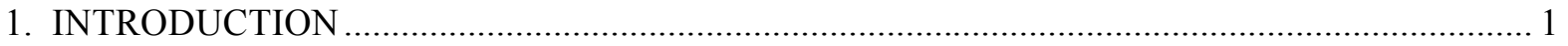

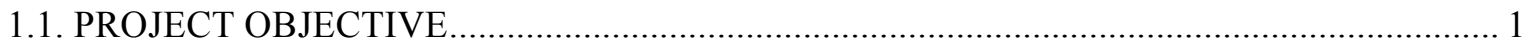

1.2 PROJECT BACKGROUND ..................................................................................... 1

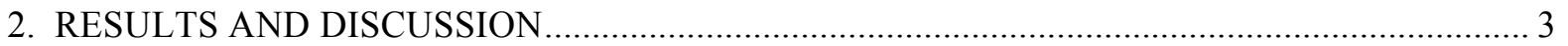

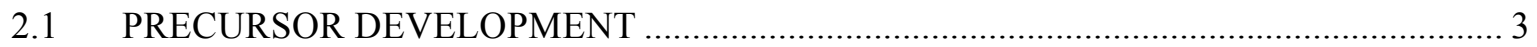

2.2 SCALEUP AND ENHANCEMENT OF ADVANCED CONVERSION PROCESSES ...... 9

2.2.1 Processing Modeling as Supported via Dielectric Measurement System .................. 9

2.3 MICROWAVE-ASSISTED-PLASMA (MAP) SYSTEM SCALING .......................... 11

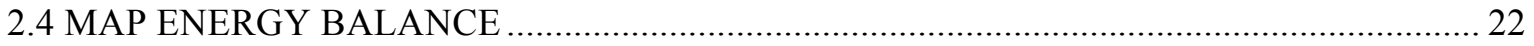

2.5 ALTERNATIVE PROCESS INVESTIGATIONS .................................................. 22

3. ACCOMPLISHMENTS AND CONCLUSIONS ......................................................... 29

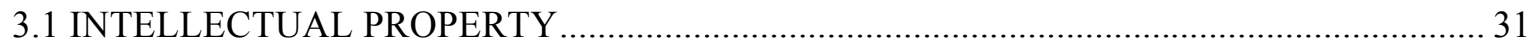

3.2 PUBLICATIONS AND PRESENTATIONS ............................................................ 31

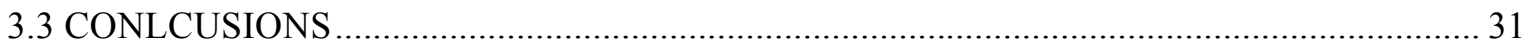

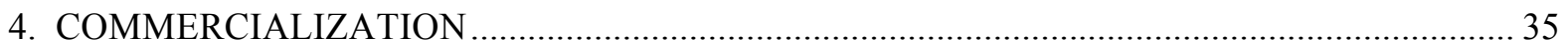

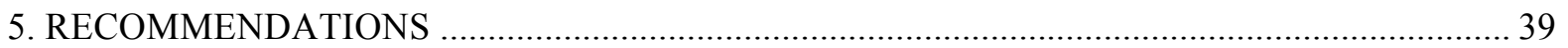




\section{LIST OF FIGURES}

Fig. 1. TGA thermogram of a crosslinked polymer precursor indicating the need for secondary functionalization.

Fig. 2. Stress-strain profiles of a functionalized polymer precursor. …............................................. 5

Fig. 3. Stress-strain profiles of a neat and stretched polymer precursor. ......................................... 5

Fig. 4. Thermomechanical thermograms of two different functionalized precursors. ........................ 6

Fig. 5. Thermomechanical thermograms of a functionalized precursor at different applied stress

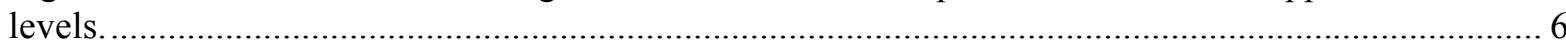

Fig. 6. SEM images of fracture surfaces of fibers with the best tensile strength for a sample (process

ID 110606T4) carbonized under low tension (a) and the same precursor sample (process ID

110616T1) carbonized under high tension (b). ..................................................................... 7

Fig. 7. ORNL diagnostic measurement cavity with associated heating system................................. 9

Fig. 8. The loss tangents as a function of temperature of selected materials that are of interest in

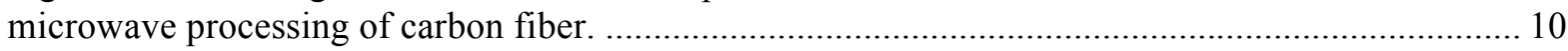

Fig. 9. The current density shown inside the plasma [processing] zone for two different values of "plasma" conductivity. As the conductivity goes up,the penetration of the microwaves into the plasma is reduced which also reduces the amount of processing near the center.......................................... 11

Fig. 10. Maximum temperature of quartz tube and of gas at outlet vs. gas inlet pressure................... 11

Fig. 11. Commercial filter-trap installed on the MAP vacuum foreline. ....................................... 12

Fig. 12. Geometry of the tows within the vacuum tube. The earlier configuration (a) had the tows oriented vertically while most recently this was changed to a horizontal orientation (b). The red dotted circles are the extent of the old, smaller tube used for 3-tow experiments in 2011. The dashed circle prescribes the radius of the outermost tows prior to the 5 -tow experiments............................ 13

Fig. 13. Intra-tow tension differences in oxidized PAN feedstock. Part of the tow is under very little

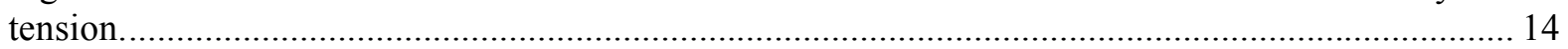

Fig. 14. Tow orientations within MAP applicator. (a) Orientation in pre-2012 multi-tow experiments. Heating is very uneven. (b) Attempted tow orientation in 3Q2012 campaign. (c) Tow orientation after applicator rotation for 5-tow experiment in early FY 13. ............................................................. 14 Fig. 15 . (a) The MAP line after rotating the applicator. Microwave coupling is now from below. (b) The MAP line exit feed-throughs are shown fully strung with five tows in the horizontal configuration 14

Fig. 16. Time trace of 5-tow, 2+ hour milestone run. Shown are the forward and reflected power, the nominal maximum vacuum tube temperature, the outlet gas temperature, and the tube gas pressure. At $\mathrm{t}=17$ minutes, the microwave generator tripped.

Fig. 17. Mechanical properties from selected MAP runs. The orange, red and cyan points are from three 5-tow runs. Fiber properties in the shaded region meet or exceed programmatic targets. Large variance is due to single-filament mechanical testing................................................................. 16 Fig. 18. Mechanical properties and relative positions of a single 5-tow MAP run for the horizontal configuration (left) and the "smiley-face" configuration (right).................................................... 17 Fig. 19. Proposed multi-cavity MAP applicator. Each cavity should be nominally uncoupled from the others, unlike the plasma zones in the side-wall coupled applicator................................................. 18

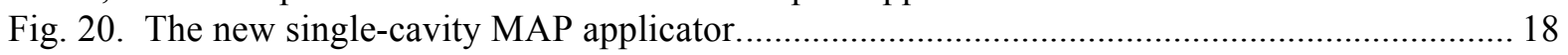

Fig. 21. A plasma test with a single tow in the v4 MAP applicator................................................ 19

Fig. 22. The triple-cavity MAP applicator shown here with $\mathrm{rf}$ and UV shielding. .......................... 20

Fig. 23. The tow configurations for $450 \mathrm{k}$ tows and a single large $250 \mathrm{k}$ tow............................... 20

Fig. 24. Mechanical properties of MAP-processed carbon fiber for selected runs. MAPv4.2 corresponds to a dual-cavity system; MAPv4.3 is the triple-cavity system. The properties for multitow runs are averaged over all tows. 
Fig. 25. New ceramic filter system in line with primary vacuum line ..........................................21

Fig. 26. Energy and mass inputs and outputs for the MAP process. ................................................22

Fig. 27. Atmospheric pressure plasma torch operating with oxidized tow.......................................23

Fig. 28. Temperature profiles with respect to the axial distance from the plasma torch nozzle..........24

Fig. 29. Atmospheric pressure plasma torch operating with an oxidized tow under inert atmosphere.

The copper coil at the upper center is a heat exchanger for the "bell jar" enclosure...........................24

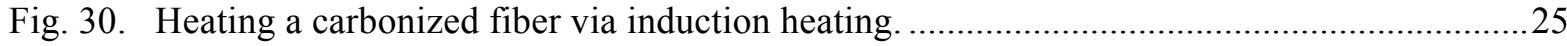

Fig. 31. Atmospheric plasma carbonization experimental setup at ReMaxCo................................26

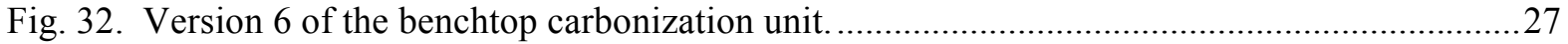

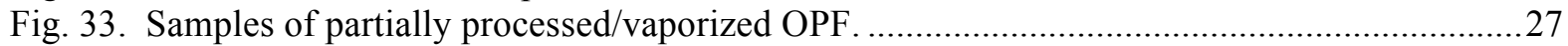

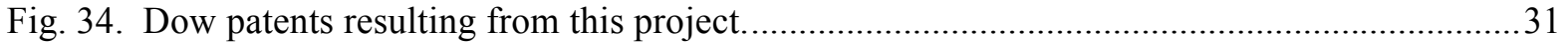




\section{ACRONYMS}

$\begin{array}{ll}\text { AMO } & \text { Advanced Manufacturing Office } \\ \text { CFTF } & \text { Carbon Fiber Technology Facility } \\ \text { COEE } & \text { Center of Energy Excellence } \\ \text { CPEC } & \text { Close-Proximity Electromagnetic-Heating for Carbonization } \\ \text { CRADA } & \text { Cooperative Research and Development Agreement } \\ \text { DOE } & \text { Department of Energy } \\ \text { EERE } & \text { Office of Energy Efficiency and Renewable Energy } \\ \text { EMI } & \text { Electro-Magnetic Interference } \\ \text { FTIR } & \text { Fourier Transform Infrared Spectroscopy } \\ \text { GPa } & \text { GigaPascal } \\ \text { ICP } & \text { Inductively Coupled Plasma } \\ \text { Ksi } & \text { Kilopounds per Square Inch } \\ \text { KW } & \text { KiloWatt } \\ \text { LCCF } & \text { Low Cost Carbon Fiber } \\ \text { LLDPE } & \text { Linear Low Density Polyethylene } \\ \text { MAP } & \text { Microwave-assisted Plasma } \\ \text { Msi } & \text { Million Pounds per Square Inch } \\ \text { OPF } & \text { Oxidized PAN Fiber } \\ \text { ORNL } & \text { Oak Ridge National Laboratory } \\ \text { PAN } & \text { Polyacrylonitrile } \\ \text { PO } & \text { Polyolefin } \\ \text { RF } & \text { Radio Frequency } \\ \text { SBIR } & \text { Small Business Innovation Research } \\ \text { TGA } & \text { Thermal Gravimetric Analysis } \\ \text { US } & \text { United States } \\ \text { VTP } & \text { Vehicles Technology Program } \\ & \end{array}$




\section{ACKNOWLEDGEMENTS}

This project was funded by the US Department of Energy (DOE), Office of Energy Efficiency and Renewable Energy (EERE) Advanced Manufacturing Office (AMO) and partially co-funded by the Vehicle Technologies Program (VTP) for the advanced conversion portion. This advanced materials project was managed as CPS Agreement Number: 20930. The lead organization was the Oak Ridge National Laboratory (ORNL) in partnership with the Dow Chemical Company and the Michigan Economic Development Corporation. The project duration was from April 1, 2010 through March 31, 2014. Work on process modeling was supported by Stephanie Diem and work on alternative carbonization techniques was guided by Truman Bonds of ReMaxCo and Philip Pesavento at ORNL. The experimental work was supported by a large number of individuals at ORNL, Dow, and ReMaxCo. Programmatic support by ORNL and DOE staff members aligned with AMO and VTP and related partnership organizations is greatly appreciated. 


\section{EXECUTIVE SUMMARY}

The overall objective of the collaborative research performed by the Oak Ridge National Laboratory (ORNL) and the Dow Chemical Company (Dow) was to develop and establish pathways to commercialize new carbon fiber precursor and conversion technology. This technology is to produce alternative polymer fiber precursor formulations as well as scaled energy-efficient advanced conversion technology to enable continuous mode conversion to obtain carbonized fibers that are technically and economically viable in industrial markets such as transportation, wind energy, infrastructure and oil drilling applications. There have been efforts in the past to produce a low cost carbon fiber. These attempts have to be interpreted against the backdrop of the market needs at the time, which were restricted to military aircraft and high-end aerospace components. In fact, manufacturing costs have been reduced from those days to current practice, where both process optimization and volume production have enabled carbon fiber to become available at prices below $\$ 20 / \mathrm{lb}$. However, the requirements of the lucrative aerospace market limits further price reductions from current practice. The approach taken in this project is different because specific industrial applications are targeted, most specifically wind turbine blade and light vehicle transportation, where aircraft grade carbon fiber is not required. As a result, researchers are free to adjust both manufacturing process and precursor chemistry to meet the relaxed physical specifications at a lower cost. This report documents the approach and findings of this cooperative research in alternative precursors and advanced conversion for production of cost-effective carbon fiber for energy missions.

The project team made significant accomplishments in advancing both the development of polyolefinic (PO) formulations and fibers for use as carbon fiber precursors as well as the development of advanced carbonization techniques for conversion of stabilized precursors into carbon fiber. In going into this project, ORNL had demonstrated the basics of both approaches at the bench scale but the results at that point were very inconclusive as to capability to scale either approach to commercially viable technologies. At project initiation, ORNL had demonstrated the sulfonation approach for stabilizing very small samples of common polyolefinic precursors melt spun under contract to ORNL specifications and then converted into carbon fiber. Although ORNL had advanced the mechanical properties significantly, they were short of automotive minimum targets. All of the spinning, stabilization, and conversion processes had been conducted in small batch-scale processing. Working together, Dow and ORNL evaluated a very wide selection of PO formulations from the vast array of Dow commercial and developmental product lines and selected candidates most appropriate for scaling to commercial-scale production of carbon fiber. Many of these formulations were then spun into fiber at Dow and more fully evaluated in stabilization, carbonization, and mechanical testing by both organizations. The bench-scale batch approach to sulfonation developed by ORNL was converted by the team to continuous processing at Dow that, while still at relatively small-scale, facilitated projection of requirements for the complete process at commercial scale for economic analysis. More importantly the project team developed key understanding of formulation/processing relationships necessary to meet the minimum performance targets and move towards performance between the minimum required for introduction and goals that should make the lower cost forms more attractive in other application areas.

As the team met the minimum mechanical property targets in the final project year as specified by the US automotive industry, Dow was completing its updated evaluation of the economic and technical tradeoffs necessary to make a commercial launch decision. The updated economic projections at Dow indicated that although cost benefits were expected, the projected level of the benefits was likely not sufficient to entice the large capital investment projected for commercial-scale implementation of the baseline sulfonation process utilized for much of this work. Although minimum programmatic property targets were achieved and the precursor development work was on schedule to meet final 
property milestones, alternative processing routes are desirable to mitigate investment risks. Therefore, the precursor development work was placed on hold pending resolution of Dow assessment of alternatives and plans forward in this area.

ORNL had demonstrated the basics of the microwave assisted plasma process earlier in initial feasibility work funded by the DOE Vehicles Technology Program (VTP). During this feasibility work, the process was primarily demonstrated with a single tow with limited processing of up to three tows simultaneously as a maximum. The process was typically stable for only a few minutes at a time, adequate enough to produce only a few feet of converted carbon fiber for testing. Process durability defined as being able to run continuously stable over at least several hours with repeatable properties from tow-to-tow and along each tow were acknowledged as being short falls that needed to be overcome on the pathway to commercialization along with a proven ability to process more than a single tow in each module. This project tackled these issues and demonstrated that these goals can be met.

The goal of the advanced conversion portion of this project is to demonstrate the technology necessary to move to the next stage of scaling to commercialization. Based on feedback from carbon fiber manufacturing insiders and the end user community, a key target was to demonstrate technology readiness levels commensurate with having the information necessary to move forward to preproduction scale consistent with production capabilities recently introduced at the ORNL Carbon Fiber Technology Facility (CFTF) in hand at the conclusion of the project. This capability is defined by 24 tows of 24,000 filaments ( $24 \mathrm{~K}$ tows) running at about $60 \mathrm{in} / \mathrm{min}$ or similar combinations. The project team with sponsor concurrence defined the deliverable evidence of meeting this goal as production of a technical specification for equipment to go into CFTF for producing carbon fiber for evaluation purposes (both equipment as well as carbon fiber product) at this scale.

Going into the project, one of the key issues inhibiting scaling was lack of capability to distribute the energy as needed in the progression of fiber in going from a non-conductive oxidized (or stabilized) precursor to completed carbonized and relatively conductive carbon fiber. Related to this difficulty was the need to evenly distribute the energy across tows being processed concurrently. Early in this project the team established capability to measure dielectric properties of fibers at various stages of conversion as a function of the temperature environment. Dielectric properties influence coupling in an electromagnetic field, which allows basic modeling of the process. Although the dynamic nature of this process in combination with the complexity of manipulating the plasma and microwave heating mechanisms make this a very complicated problem suitable for a large research project in itself, the team was able to develop and utilize models to assist with hardware development. Using the modeling as well as materials and processing data experiments, the team essentially optimized the original Microwave-assisted Plasma (MAP) process for accomplishing both low and high temperature carbonization processes (in conventional thermal processing these tow processes are typically performed in discrete steps in totally separate furnaces) for up to 5 tows up to about $48 \mathrm{in} / \mathrm{min}$.

Although the team was continuing to make progress with the baseline MAP approach, it was recognized the gains were becoming much more incremental in nature and that alternatives might make this process more attractive. Early on the team acknowledged that the low temperature carbonization with much higher effluent generation would be the greatest challenge for the MAP process operating at the low pressures required to generate and maintain a stable plasma. Several approaches were evaluated and a team led by long-term partner ReMaxCo demonstrated basic feasibility for what is described as Close-Proximity Electromagnetic-Heating for Carbonization (CPEC) that works exclusively in the low temperature portion of the carbonization. This process was identified as having great potential, but was judged to be too immature for concentration in the final demonstration phase of this project. With this verdict, work on alternative low temperature 
carbonization process alternatives was deferred for later consideration.

During this time frame an alternative technique for dividing and controlling energy deposition in multiple chambers was proposed and evaluated. Initial work was even more promising than expected, and this technique was expanded to demonstrate its effectiveness over three-chamber deposition with a single cylindrical processing reactor. With this configuration the team was able to produce converted carbon fiber at $60 \mathrm{in} / \mathrm{min}$.

Another issue of importance to scaling and ultimate energy efficiency is effluent handling. At each point of scale-up in going to more tows and higher speeds the generation of effluent has increased significantly. At each step increase, new approaches were implemented. While the team will not claim this issue has been resolved, it is felt that significant improvements were implemented at several points to get to the next step and that standard industry approaches in production may be capable of being modified for the advanced processes with proper attention to pumping issues. There is no reason to believe that this process generates any more effluent than the standard thermal processing and the plasma may possibly have a somewhat mitigating effect. However, effluent handling will be a key issue to tackle in the scaling to the next level of production.

During the course of the project the team did demonstrate capability of the process to be operated for extended periods of time. Although it is believed that a key inherent advantage of the MAP (and the CPEC) process is the capability to stop and start rapidly, greatest efficiencies will come from continuous operations. Two-hour and eight-hour continuous operating milestones were achieved with little difficulty during this project and the team saw no signs of instabilities or that operational limits were being approached.

The project team believes the advanced carbonization technology is capable of meeting the previously outlined targets for scaling to the next level. The deliverable project specifications for scaling the carbonization system to the 25-ton-per-year CFTF production level have been drafted and can be shared with interested parties. However, due to the specification having both export controlled processing information and background for potential patent applications, this information cannot be released publicly.

ORNL believes there is merit in advancing both the polyolefinic precursor development and the advanced carbonization technologies. The cost of the PO base polymer, cost of the melt spinning and carbonization processes, and the resulting high carbon yield all tilt in favor of PO-based materials being attractive as carbon fiber precursors, if an acceptable stabilization approach can be found. Due to questions concerning the economical attractiveness of scaling the sulfonation process that was the focus of this effort, follow-on efforts should focus on alternative methods such as a gas-phase process or other means for rapidly stabilizing the PO material with less environmentally challenging materials than utilized in the baseline sulfonation process. Alternative approaches might be initially explored through smaller and more basic projects such as Small Business Innovation Research (SBIR), incubator funding, or seed money efforts. Much of materials selection and carbonization process development accomplished in this project should be applicable to follow-on work should a potentially attractive stabilization process be identified.

The team strongly recommends moving the advanced carbonization process along the next step(s) towards commercialization. Prior to actually designing and building modules for the higher production levels consistent with CFTF, the team should explore approaches for running at significantly higher line speeds. Preliminary industry feedback is that targeting very high line speeds $(>250 \mathrm{in} / \mathrm{min}$ ) might be more attractive to the industry than meeting the previously identified preproduction rates at current CFTF line speeds. Although shorter actual residence times for 
carbonization have been shown in this work, approaches for 1) running at higher line speed will likely require adding cavities in a series versus scaling the new baseline to higher production levels while 2) matching CFTF line speed requires adding processing modules in parallel. It is also likely that the alternative CPEC low temperature carbonization process will facilitate operations at higher speeds since operating at atmospheric pressure levels makes effluent removal somewhat simpler. Because this technology is much less mature than the new baseline process, scaling of the low temperature carbonization process will need to quickly catch up and surpass current processing levels for the baseline to meet the ultimate scaling targets. The team recommends working on issues associated with adding additional cavities in series for the high temperature carbonization process utilizing materials that have already been processed through a representative low temperature carbonization process in parallel with verifying that the CPEC process can be scaled to match the current baseline. The baseline low-pressure approach will be required for the high temperature process regardless of the success for the atmospheric pressure CPEC approach in low temperature carbonization. With these results in hand the complete carbonization process can be more effectively scaled to meet the pre-production level. The team believes continuing to strengthen teaming relationships with carbon fiber producers and carbon fiber production equipment manufacturers will enhance probability both of technical success in scaling and longer-term success in moving this technology into commercialization. 


\section{INTRODUCTION}

This project was divided into two areas:

1) Precursor development, and

2) Scale up and enhancement of advanced conversion processes

\subsection{PROJECT OBJECTIVE}

The production cost of commercial grade carbon fiber is about equally split between raw materials and the conversion process; therefore it is necessary to attack both materials and conversion costs to meet the cost targets specified by the US DOE. The specific objectives for this work included:

1. Develop polyolefin precursors

a. Commercial polyolefins and blends

b. Molecularly designed polyolefin fiber (such as that based on polyethylene or polypropylene) that can be crosslinked prior to carbonization

2. Demonstrate accelerated crosslinking route(s) for chemically functionalized precursor fibers

3. Obtain carbonized fiber from the inert precursors by applying optimal conventional and advanced conversion parameters

4. Characterize the carbon fiber and show that the mechanical properties are satisfactory for future product development in composite industries

5. Scale-up efforts will leverage the pilot line currently under development at ORNL

6. Evaluate materials, sealing, and atmospheric pressure solutions

7. Estimate energy requirement for various scales

8. Determine preferred microwave/plasma parameters and profiles

9. Scale to $\geq$ five large tows

10. Determine long-term, continuous operability

11. Develop design specifications to facilitate scale-up of this approach for implementation in the ORNL Carbon Fiber Technology Facility

At the conclusion of this project, objectives were for the team to have identified and demonstrated one or more formulations for an alternative carbon fiber precursor and the spinning and conversion protocols necessary to manufacture a carbon fiber that meets mechanical property and economic targets identified as adequate for production scale up. The team would have developed equipment and process knowledge adequate to produce specifications for the scale-up of an advanced carbonization process for demonstration in the ORNL Facility. These accomplishments represent a significant step in the commercialization process, bridging a large portion of the gap between benchscale research and pre-production-scale demonstration.

\subsection{PROJECT BACKGROUND}

ORNL is a world leader in the development of new materials and processes for the manufacture of carbon fibers. For close to a decade, ORNL has been developing alternative precursors, advanced conversion processes, and other technology aimed at reducing the cost of commercial grade carbon fibers. Alternative precursors under development by ORNL with US Department of Energy (DOE) sponsorship include textile grade polyacrylonitrile-based (PAN) fibers, lignin-based precursor fibers, and polyolefin-based precursor fibers. ORNL possesses world-class expertise and unique physical resources for carbon fiber research and development. 
The Dow Chemical Company is the world's largest manufacturer of polyolefin resin, and a world leader in chemistry, materials science, engineering and manufacturing excellence. For example, Dow Chemical Company's ASPUN ${ }^{\mathrm{TM}}$ and ENGAGETM polyolefin fibers are commercially available and used today in a range of products, but existing forms are not appropriate for use as carbon fiber precursors. Development of new, value-added products targeting low cost carbon fiber manufacture from polymers designed with the cost and performance requirements of carbon fiber in mind will generate additional, sustainable revenue for the Company.

Dow Chemical Company objectives were to accelerate ongoing developmental efforts to achieve commercialization within five years. This will require a dramatically accelerated research, development, demonstration, and deployment program. This project addresses the accelerated research with detailed fundamental understanding, development, and demonstration of advanced conversion processes and polyolefin-based carbon fiber precursor production.

Polyolefin fibers, such as polyethylene and polypropylene can easily be melt-spun. Dow Chemical Company utilized both Michigan Center of Energy Excellence (COEE) and in-kind contributions to invest in polymer precursor development, including building out fiber spinning capability in Michigan. This program was initiated with an open consideration to all types of polyolefinic fiber formulations and spinning techniques that are available to allow for the development of a wider class of low-cost polymer precursor fiber. After selecting a preferred family of material chemistries, the project participants down-selected to the most cost-effective means of preparing the precursor fiber and converting the precursor into carbon fiber.

Conventional conversion employs a thermal pyrolysis process that is slow, energy intensive, and space intensive. This advanced conversion portion of this project is based upon a discovery by the principal investigator that microwave energy could likely be used as a means to rapidly convert polyacrylonitrile precursor fibers into finished carbon fibers while potentially reducing energy usage substantially. Early in the project, the researchers determined that the best approach was to use a combination of single-frequency microwaves and plasma, or microwave-assisted plasma (MAP), to carbonize and graphitize oxidized PAN fibers. MAP processing feasibility was demonstrated in a batch reactor. Continuous operation was then demonstrated in a low-speed single-tow reactor, which was subsequently scaled to achieve high-speed single-tow operation, and finally three-tow operation. The researchers demonstrated a significantly reduced residence for the advanced process versus the conventional baseline.

Federal funding for this integrated project addressing alternative precursors and advanced conversion was provided by the U.S. Department of Energy's Advanced Manufacturing Office and Vehicle Technologies Program and activities were executed via a Cooperative Research and Development Agreement (CRADA). ORNL and Dow worked together to accelerate development and commercialization of low-cost polymer-based carbon fiber precursors and advanced conversion processes. This work built on the foundation and substantial efforts of ORNL staff that had been funded through the U.S. DOE Vehicle Technologies Program. 


\section{RESULTS AND DISCUSSION}

ORNL and Dow exchanged several visits and numerous teleconferences to define and refine specific approaches and metrics for materials of interest to this program. Based on previous ORNL work and studies conducted as part of this work, a better understanding was developed between Dow and ORNL on the underlying economics of material and process choices impacting portions of the technical approaches. This information and these discussions were utilized to develop a basic agreement on division of tasks and task responsibilities. Contracts personnel at both organizations were apprised of this general agreement and consulted as to the best mechanism or mechanisms for formalizing the agreement into the appropriate legal documentation. Although not required by either organization or funding agencies, it was determined that a Cooperative Research and Development Agreement (CRADA) was indeed the appropriate format for the formal agreement and the CRADA document was completed in October of 2010.

As has been the case for related work in carbon fiber development, ORNL has found an effective working arrangement to be establishment of a critical leadership core of permanent researchers with significant background and experience in this area (carbon fiber alternative precursor and advanced conversion development) to be augmented with postdoctoral researchers bringing specific skills to this project. Through detailed analysis of project requirements and extensive recruiting efforts, most of the defined needs for these postdoctoral researchers were identified and these personnel were relocated to ORNL to support this activity. Likewise, a need for rheology evaluation via extrusion was defined and equipment necessary for this work was specified for procurement. Effort was undertaken to develop work plans for a subcontractor with extensive background and experience in the area of atmospheric plasma processing in carbon fiber oxidation to extend on-going collaboration with ORNL to work in the carbon fiber carbonization process.

Significant development and demonstration in precursor and advanced conversion process development represent a significant step in the commercialization process bridging a large portion of the gap between initial technology development and feasibility demonstration and the scale-up to preproduction volumes and operational time periods necessary to justify market entry. Activities and accomplishment in these areas are described as follows:

\subsection{PRECURSOR DEVELOPMENT}

Polyolefin fibers, such as polyethylene and polypropylene can easily be melt-spun. However, a wider variety of polymers and copolymers are capable of being solution-spun (wet-spinning), and may not be amenable to melt spinning. Any polymer that can be put into solution can, in principle, be prepared by solution-means into a fiber. Therefore, plans were to initiate this program with an open consideration to all types of fiber spinning that are available to allow for the development of a wider class of low-cost polymer precursor fiber. Then, after selecting a preferred family of material chemistries, the most cost-effective means of preparing the precursor fiber were to be down-selected. A protocol for screening polyolefin precursor composition suitable for carbon fiber research and development by the team was developed and processing parameters to obtain precursor fibers from selected compositions determined. Solutions for precursor fiber crosslinking were proposed and feasibility of specific candidates producing adequate material properties evaluated. As necessary, accelerated crosslinking route(s) for chemically functionalized precursor fibers were developed culminating in demonstrated production of carbonized fiber from cross-linked precursors following conventional and microwave-assisted plasma conversion processes. 
A key objective of this work was to develop technologically designed fiber-forming precursor materials. Parameters for the precursor screening metrics were identified during initial project work. Precursor screening parameters include melt-processability of the precursor resin (in terms of meltflow index), crystallinity in filament, film, or bulk material, and ability to convert the precursor to thermally stable form. ORNL researchers worked with Dow to develop this selection procedure. Characterization of a different type of cross-linked precursor (common for high voltage cable insulation) was conducted. A representative thermal gravimetric analysis (TGA) thermogram of the material is displayed in Fig. 1. The material appeared non-carbonizable. Chemical functionalization is necessary. Because of CRADA protection, details of various identified precursor candidates are not disclosed.

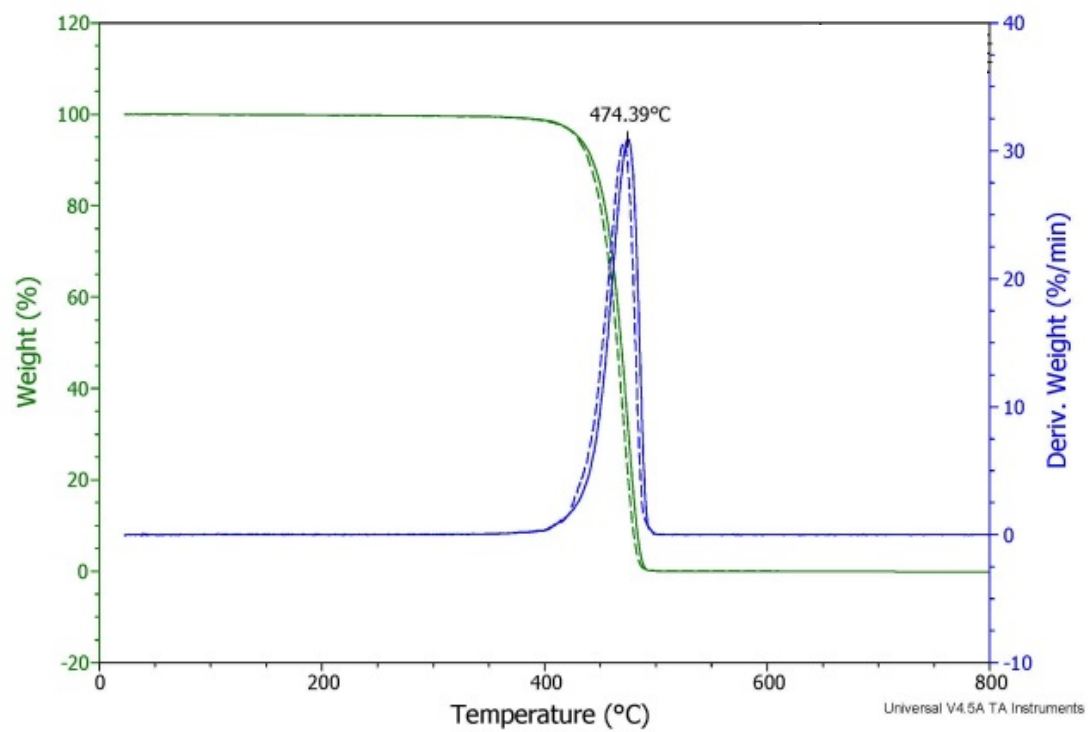

Fig. 1. TGA thermogram of a crosslinked polymer precursor indicating the need for secondary functionalization.

Another key objective of this work was to find processing parameters for functionalized precursors. Precursor fibers at various degrees of functionalization were investigated and the thermo-mechanical properties of those fibers studied. A protocol for establishing fiber stress-strain profiles at various temperatures was developed to understand the role of functionalization and dimensional stability during the conversion process of these new types of precursors. Stress-strain profiles of representative functionalized and neat samples are shown in Fig. 2. These data are important to establish the conversion (heat treatment and carbonization) protocol. With increase in temperature the modulus of functionalized fiber decreases but ultimate elongation is not significantly affected in comparison to neat fibers. This indicates that the functionalized fibers have higher tolerance to heat treatment compared to that of the neat fibers. Thus, this functionalization offered dimensional stability in the precursor and such stability is needed prior to carbonization. 


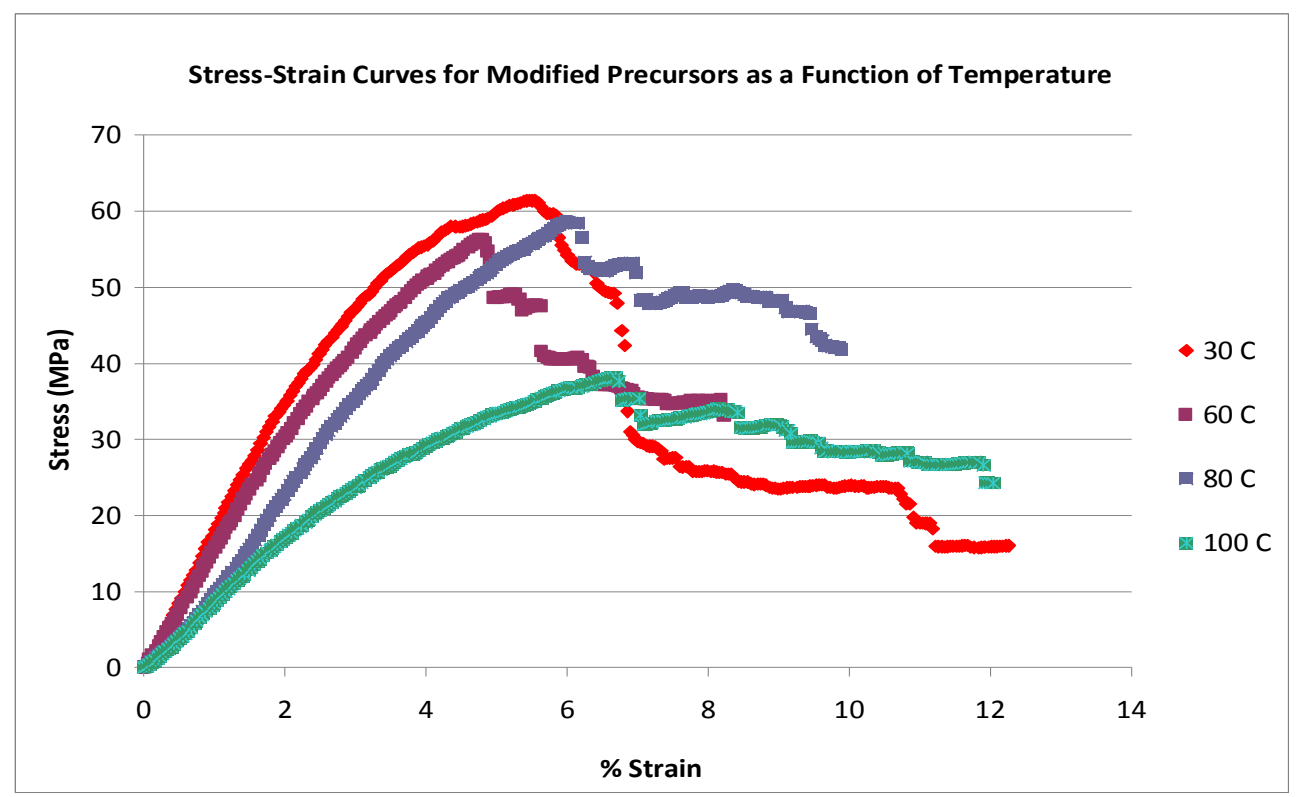

Fig. 2. Stress-strain profiles of a functionalized polymer precursor.

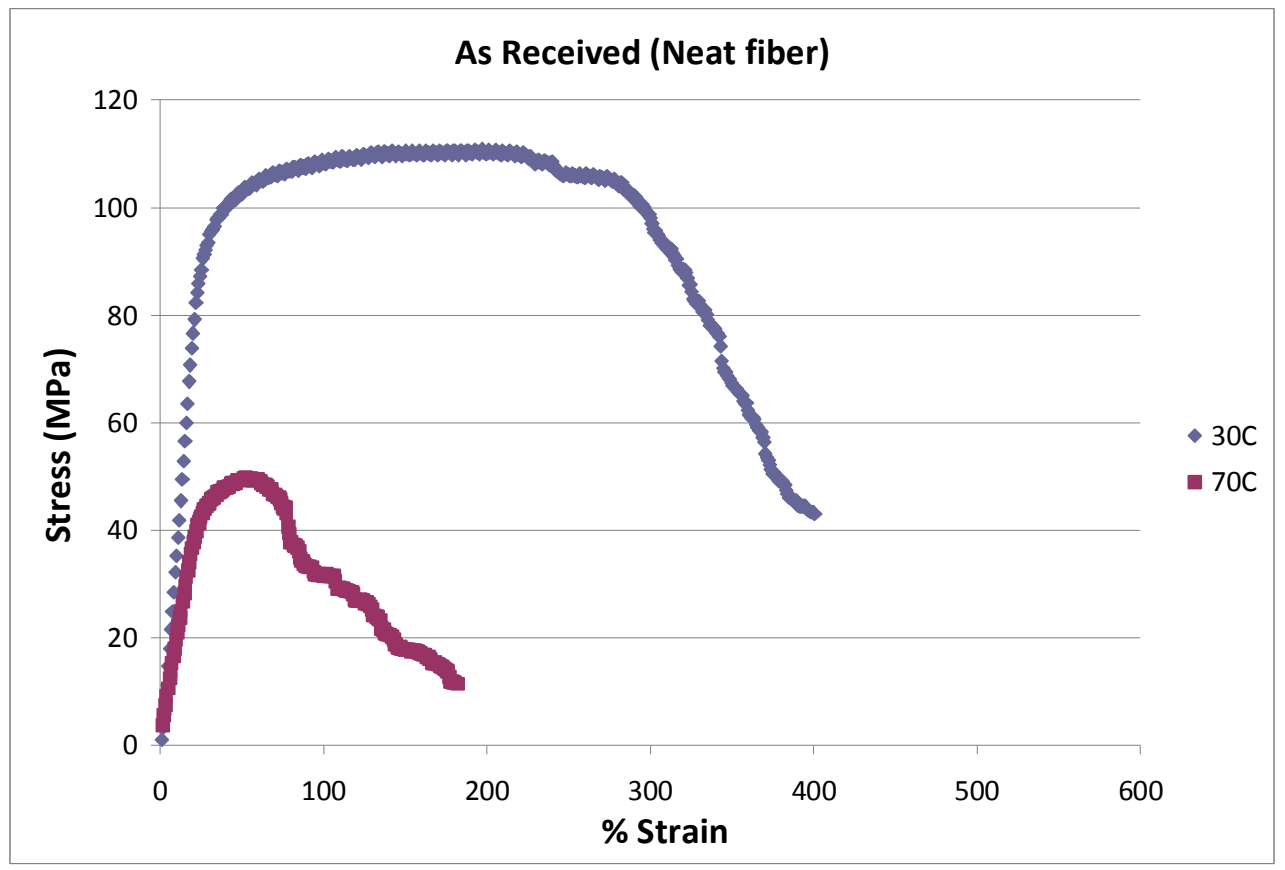

Fig. 3. Stress-strain profiles of a neat and stretched polymer precursor.

Originally, it was intended to initiate this program with an open consideration to all types of fiber spinning (solution and melt-spinning) that are available to us to allow for the development of a wider class of low-cost polymer precursor fiber. However, based on early results and availability of wide varieties of melt-processable precursor, it was decided to focus on melt-processable plastics unless unforeseen limitations downstream were uncovered. The criteria for screening these polyolefin precursors suitable for carbon fiber research and development was set up based on initial conversion results from commercially available polyethylene fibers and tentative processing parameters to obtain precursor fibers from selected compositions determined.

Protocols for dynamic mechanical analysis of the fiber tow were established to understand the 
molecular dynamics of the neat polymer precursor and its chemically functionalized derivatives in fiber form. Similarly, protocols for thermo-mechanical analyses of a tow were developed to understand dimensional stability of the fibers before and after functionalization.

ORNL and Dow researchers selected various possible fiber forming linear polymers. Dow researchers produced fibers from three of those precursors. Protocol for characterization of the fiber tow was established earlier to understand the molecular dynamics of the neat polymer precursor and its chemically functionalized derivative in fiber form. Fig. 4 shows thermo-mechanical thermograms of two different functionalized fibers showing differences in their shrinkage behavior at different temperatures. The shrinkage level at both low and high temperature regions of one sample is better than the other. Obviously, one is dimensionally more stable than the other one. This differential behavior is due to differences in degree of crosslinking, degree of crystallinity, and processing parameter (tension, time, etc.) during functionalization step. Therefore, it can be fairly assumed that the optimal processing parameters for different precursor candidates will be different.

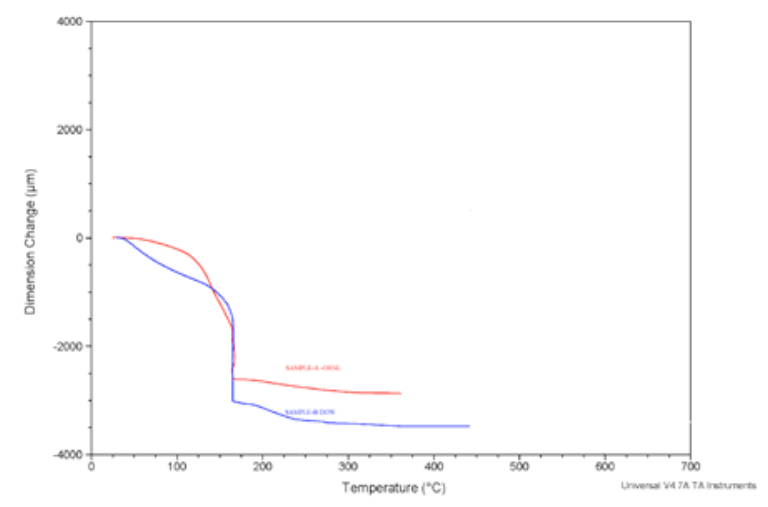

Fig. 4. Thermomechanical thermograms of two different functionalized precursors.

Thermo-mechanical analysis was used to determine convertibility of treated precursor into carbonized form by applying certain tension and to identify processing parameters that enhance mechanical properties. Fig. 5 shows that fiber shrinkage decreases with increasing applied stress during thermal treatment. However, very high shrinkage stress causes tow breakage. Fiber breaks during heat treatment step at $<200{ }^{\circ} \mathrm{C}$ at high stress levels.

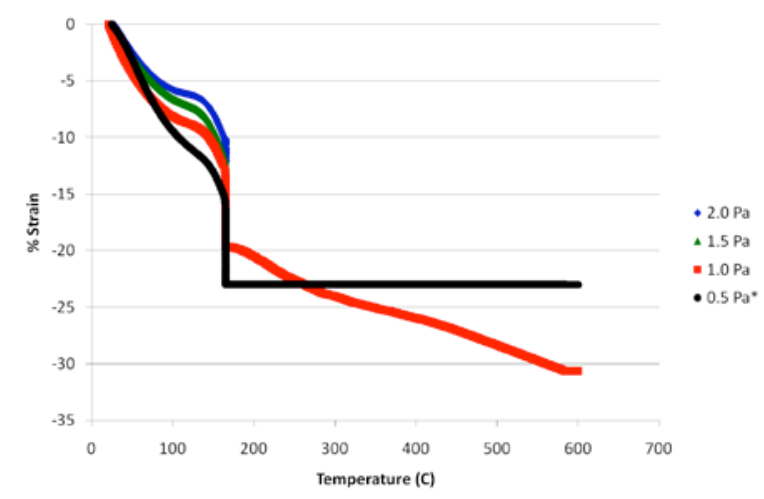

Fig. 5. Thermomechanical thermograms of a functionalized precursor at different applied stress levels.

Various characterization methods including thermal analysis tools, electron microscopy, and 
mechanical testing protocols were employed to identify better precursor fibers. Melt-spun fibers of four different precursors were obtained. Among those, two different polyolefin candidates were studied extensively and their optimal degrees of functionalization have been established. The lead precursor candidates exhibit $>60 \%$ carbon yield and demonstrate $<1 \mathrm{~h}$ processing time for the maximum possible degree of functionalization. Carbonized filaments from those precursors were also produced. Thus the baseline performance of the two precursor candidates was evaluated against the identified metrics.

Early on the research team found that with increase in the applied tension during carbonization the resulting carbon fiber's failed surface becomes rougher with multiple tear paths. At low tension during heat treatment the carbon fiber exhibits mostly smooth failure surface likely due to poor alignment of carbon microstructures along the fiber axis. Higher work of rupture as shown by multiple tear paths in Fig. 6 below is evident for the sample carbonized at significantly higher tension. This confirms that tension during carbonization, which is well known in PAN-based carbon fiber processing, has the same effect in linear low density polyethylene (LLDPE)-based carbon fiber.

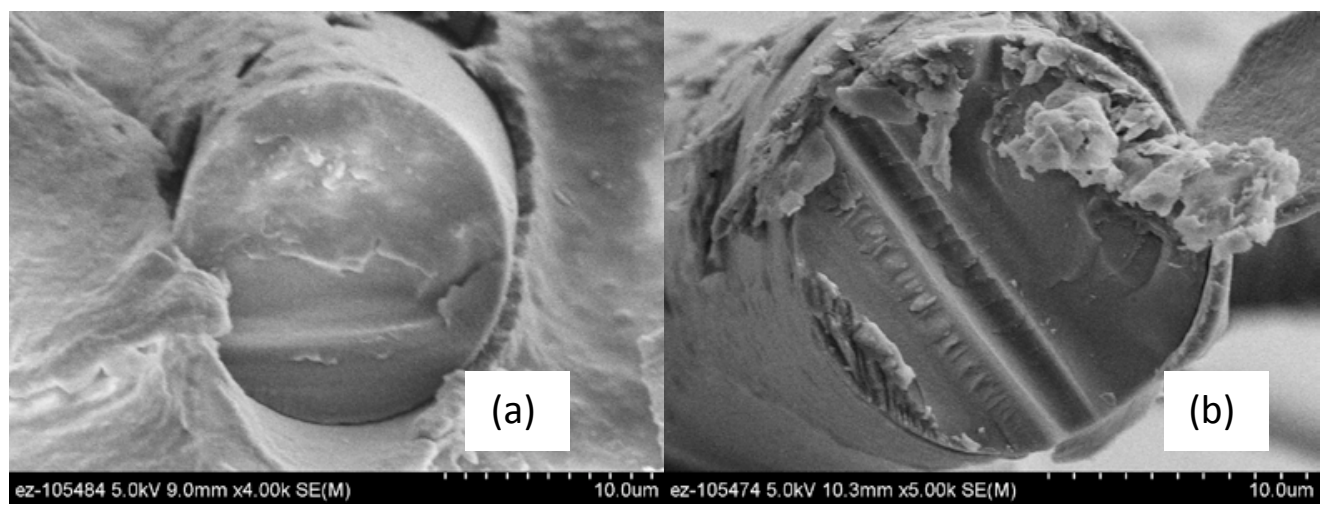

Fig. 6. SEM images of fracture surfaces of fibers with the best tensile strength for a sample (process ID 110606T4) carbonized under low tension (a) and the same precursor sample (process ID 110616T1) carbonized under high tension (b).

During the latter portion of 2011, a precursor tow free of fused filaments was sulfonated continuously and carbonized at lower temperature under constant tension inside thermo-analysis equipment. This carbonized sample has a modulus of $10 \mathrm{Msi}$ and a tensile strength of $155 \mathrm{ksi}$. The same sulfonated precursor was shipped to Dow and was carbonized to the maximum heat treatment temperature at that time, under suitable tension. This sample exhibited a modulus of $15 \mathrm{Msi}$ and a tensile strength of 154 ksi as measured by Dow. To establish consistent tensile test protocols, a round-robin testing series was conducted by the team members utilizing standard (commercial) carbon fibers. The primary difference between the ORNL process and the Dow process at the time of this work was that Dow could only carbonize samples in a batch process under a moderate heating rate whereas ORNL's continuous carbonization process could provide a rapid heating rate $\left(>100^{\circ} \mathrm{C} / \mathrm{min}\right)$. Although a slow heating rate yields higher modulus carbon fiber, it isn't economically feasible. Later on, work continued to achieve higher carbon fiber properties using conventional continuous, scalable carbonization processes. One approach was to vary the stabilization/sulfonation conditions so as to begin low-temperature carbonization during the sulfonation step. Although a similar protocol was used for testing in two different labs, more rigorous comparison of testing methods and data was done with appropriate samples as the project continued.

In a separate study, continuously-sulfonated precursor was heat treated to different temperatures to determine the effect of carbonization temperature on carbon fiber properties. In previous 
experiments, somewhat lower than expected carbon fiber properties were observed at an intermediate carbonization temperature whereas expectations were that properties would increase with increasing temperatures. It was hypothesized that the difference was mainly due to a change in furnace for higher temperature operation. In the continued study, the high-temperature furnace was used with relatively wide temperature ranges. The result was more consistent improvement in properties with higher temperatures as evident in the data discussed below. These data were obtained on a precursor that was sulfonated and carbonized in a continuous manner at ORNL.

During the last quarter of FY2012, Dow provided precursor samples in short lengths (12 inch) as well as in spools (continuous tow of length $>2 \mathrm{~m}$ ) for carbonization trials at ORNL. These samples were successfully converted and yielded targeted mechanical properties. Heat treatment at $>1800^{\circ} \mathrm{C}$ was necessary to achieve the desired modulus. Temperature optimization was the next focus with the Dow precursor candidates. ORNL had previously achieved properties of $202 \mathrm{ksi}$ tensile strength and 22 Msi modulus (at ORNL's maximum temperature process condition) with a polyolefinic precursor material produced using specialized spinning conditions not considered for focus in the project due to scalability issues. These samples were shared with Dow researchers and they confirmed the properties. With multiple sets of Dow-stabilized samples, properties of $20 \mathrm{Msi}(138 \mathrm{GPa})$ modulus and $200 \mathrm{ksi}(1.38 \mathrm{GPa})$ tensile strength were achieved. Researchers demonstrated fibrillar morphology in stabilized fibers (which was a key for mechanical property management) and that 5\% elongation was achievable. Such morphology allowed tensioning during conversion.

Work continued to build in understanding how to improve and exploit these characteristics toward higher tensile strength for automotive and potentially other applications. Subsequently Dow continued development of their own precursor composition and process control parameters. In the first half of 2013, Dow prepared the next batches of sulfonated (crosslinked and pyrolyzable) samples and those were carbonized at lower temperature than the previous runs both at ORNL and at Dow's newly installed high temperature furnace. Depending on the processing history, samples exhibited tensile strength in the range of 145-350 ksi (1.0-2.4 GPa) and modulus in the range of 16-29 Msi (110-200 GPa). A couple of batches of samples show $<1.0 \%$ ultimate elongation and those were correlated with the processing history of the fibers. Thus, while meeting a milestone for properties, a structure-property-processing relationship was developed in order to better optimize properties versus economics of the process for the specific Dow formulations.

An alternative gas-phase sulfonation-based stabilization process for polyolefin fibers was proposed by ORNL and preliminary work initiated. This processing methodology was demonstrated to provide adequate stabilization of the fibers for the subsequent carbonization step. A 6" long tow of fibers was processed using this method that does not require a contact with liquid prior to washing with deionized water. Fibers exhibited $>65 \%$ carbon yield when pyrolized at higher temperature in a thermogravimetric analyzer. Carbonization under tension had not been optimized at the conclusion of this work, as it required longer pre-processed tow than was available for key experiments. Although this work was discontinued before definitive results were obtained, a modified gas-phase reactor was designed and utilized for initial experimental work that did provide encouragement that the gas-phase approach is technically feasible for stabilization of polyolefinic precursors.

As the team was approaching the final property milestones, updated economic projections at Dow indicated that although cost benefits were expected, the projected level of the benefits was likely not sufficient to entice the large capital investment projected for commercial-scale implementation of the baseline sulfonation process utilized for much of this work. Although minimum programmatic property targets were achieved and the precursor development work was on schedule to meet final property milestones, alternative processing routes are desirable to mitigate investment risks. Therefore, the precursor development work was placed on hold pending resolution of Dow 
assessment of alternatives and plans forward in this area.

\subsection{SCALEUP AND ENHANCEMENT OF ADVANCED CONVERSION PROCESSES}

In previous work funded by the DOE Vehicle Technologies Program, ORNL researchers have carbonized a single $50 \mathrm{k}$ tow at $5 \mathrm{~m} / \mathrm{min}$, or $<1 / 2$ of conventional residence time, and $3 \times 50 \mathrm{k}$ tows at 1 $\mathrm{m} / \mathrm{min}$, or $<2 \mathrm{X}$ conventional residence time. Currently, the system was not robust and did not approach the required uptime. We proposed to begin scaling and proving the MAP carbonization process. The principal tasks of this portion of the project were:

- Evaluate materials, sealing, and atmospheric pressure solutions;

- Estimate energy requirement for various scales;

- System modeling;

- Determine preferred microwave/plasma parameters and profiles necessary to minimize residence time;

- $\quad$ Scale to $\geq$ five large tows;

- Determine long-term, continuous operability;

- Develop information necessary to produce a robust advanced technology carbonization unit to be built and operated in the ORNL Carbon Fiber Demonstration Line.

\subsubsection{Processing Modeling as Supported via Dielectric Measurement System}

A microwave cavity diagnostic for measuring dielectric properties has been constructed as shown in Fig. 7 and has been utilized to provide key information on the sensitivity of the precursor to microwave exposure as a function of the exposure temperature and degree of conversion to carbon fiber. The process control software for this system is also a candidate for use as an in situ diagnostic on the MAP line. The associated heating system allows for precise control of fiber temperatures up to approximately $700^{\circ} \mathrm{C}$. The measurement of loss tangent with respect to temperature was also used along with the modeling information to evaluate a hybrid conversion approach. An example data plot is shown in Fig. 8.

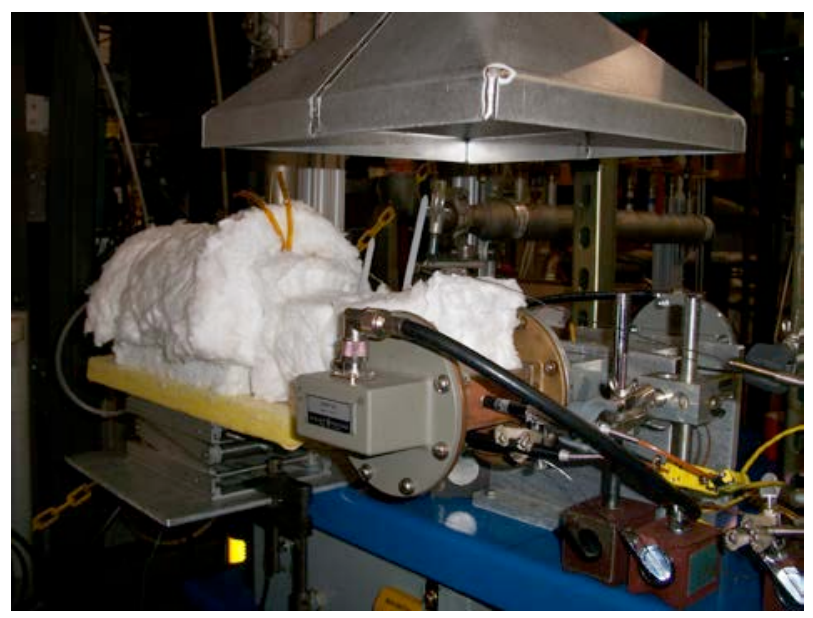

Fig. 7. ORNL diagnostic measurement cavity with associated heating system. 


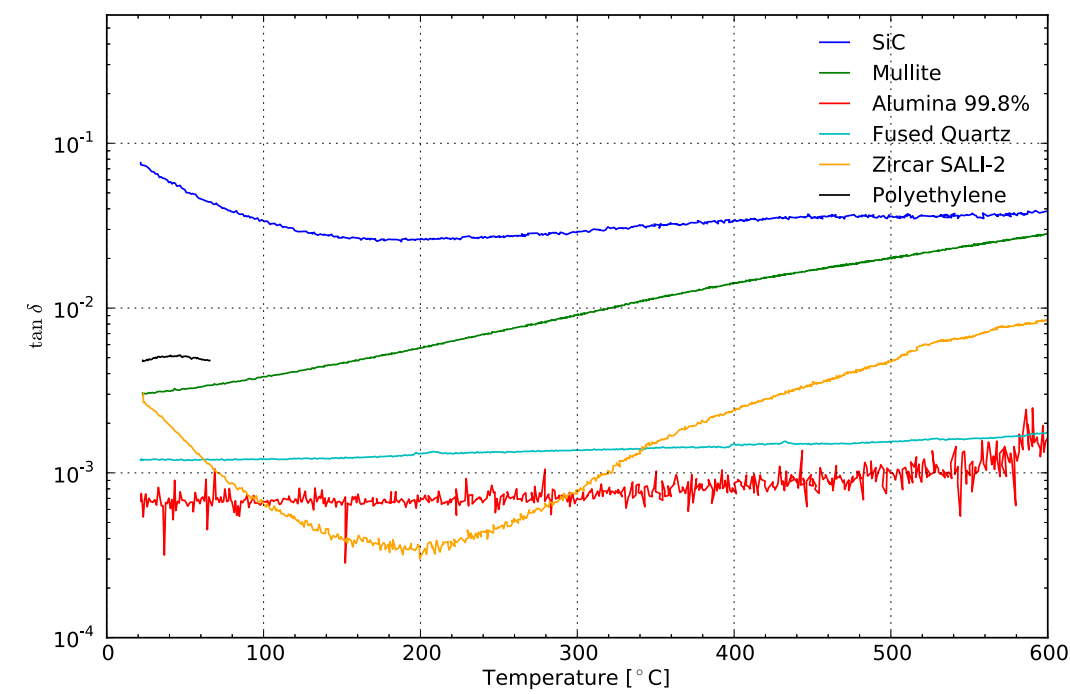

Fig. 8. The loss tangents as a function of temperature of selected materials that are of interest in microwave processing of carbon fiber.

A pair of finite element codes was used to model the MAP system. CST Microwave Studio (MWS) software is a 3D EM simulation tool that solves Maxwell's equations with the finite integration technique and finite time differencing. COMSOL is a multi-physics finite element package that can couple radio frequency (rf) and microwave power inputs with heat transfer models. A 3D model of the system was created and includes the material properties of the waveguide and quartz tube. The initial model used a uniform material with a high permittivity to simulate the plasma. The tow was assumed to be uniform and given a density, resistivity and diameter consistent with that of a carbon fiber tow post graphitization.

A Drude dielectric dispersion model was developed to more accurately model the plasma including segmenting the plasma into 1-2 inch sections to account for the non-uniformity observed in the MAP plasma. Additionally, the tow was partitioned into smaller segments to account for changes in density, resistivity, diameter and position within the quartz tube. Dielectric data from the cavity diagnostic described previously was utilized to more accurately model the tow parameters.

Given the intra-tow variance in the mechanical properties, the modeling effort was revisited to understand the causes. Treating the plasma as a poorly conducting metal, the plasma current density profile corresponding to the processing power as function of location was modeled. Fig. 9 shows two simulations of a plasma column in an applicator analogous to the MAP. At higher conductivity, the current profile changes shape considerably to become hollow. This may explain the relative lack of processing on the interior tows under certain operating conditions. 

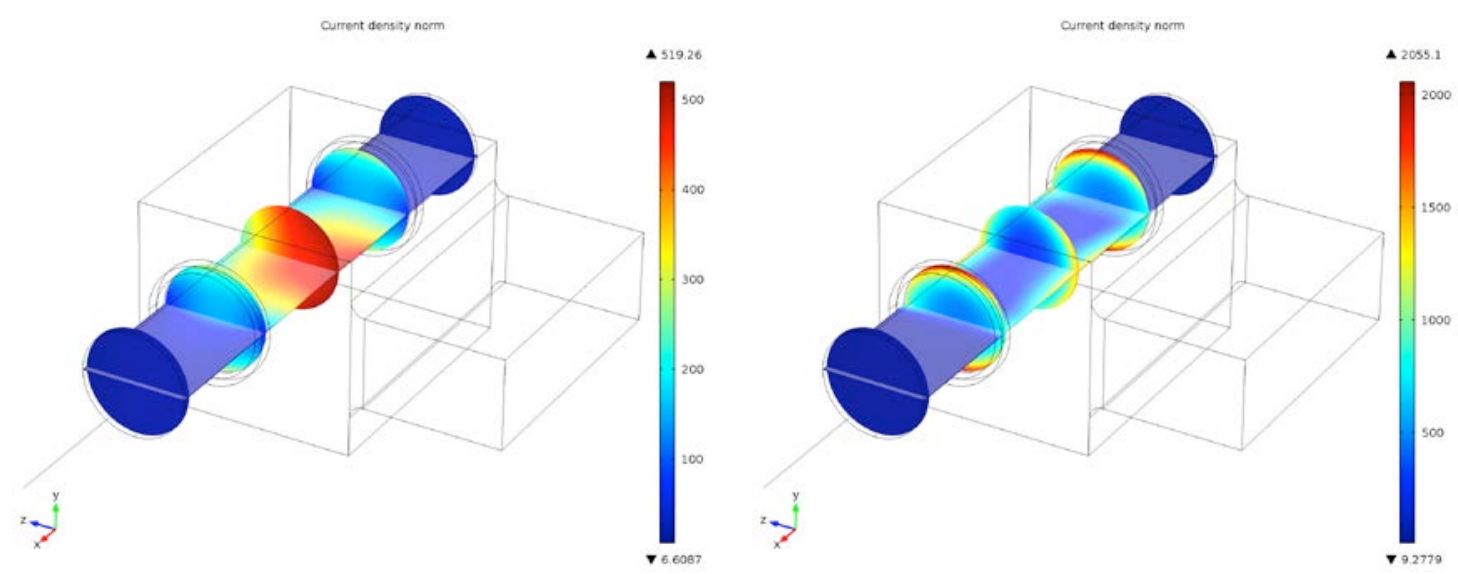

Fig. 9. The current density shown inside the plasma [processing] zone for two different values of "plasma" conductivity. As the conductivity goes up, the penetration of the microwaves into the plasma is reduced which also reduces the amount of processing near the center.

\subsection{MICROWAVE-ASSISTED-PLASMA (MAP) SYSTEM SCALING}

The baseline MAP system runs in the low-to-medium vacuum regime. This operating regime requires the use of vacuum pumps and seals that have been the focus of engineering improvements to reduce cost and enhance reliability as the system was scaled to handle more tows. A series of plasma-only baseline measurements were taken in order to develop a set of optimized operating conditions for carbonization. For example, Fig. 10 shows the how the temperature of the vacuum chamber and the working gas varies as the inlet pressure is varied. Similar experiments were done for chamber pressure and rf input power. Later experiments added the tow to determine the effect of effluent emission during carbonization as well as the modified rf absorption profile in the chamber due to the tow.
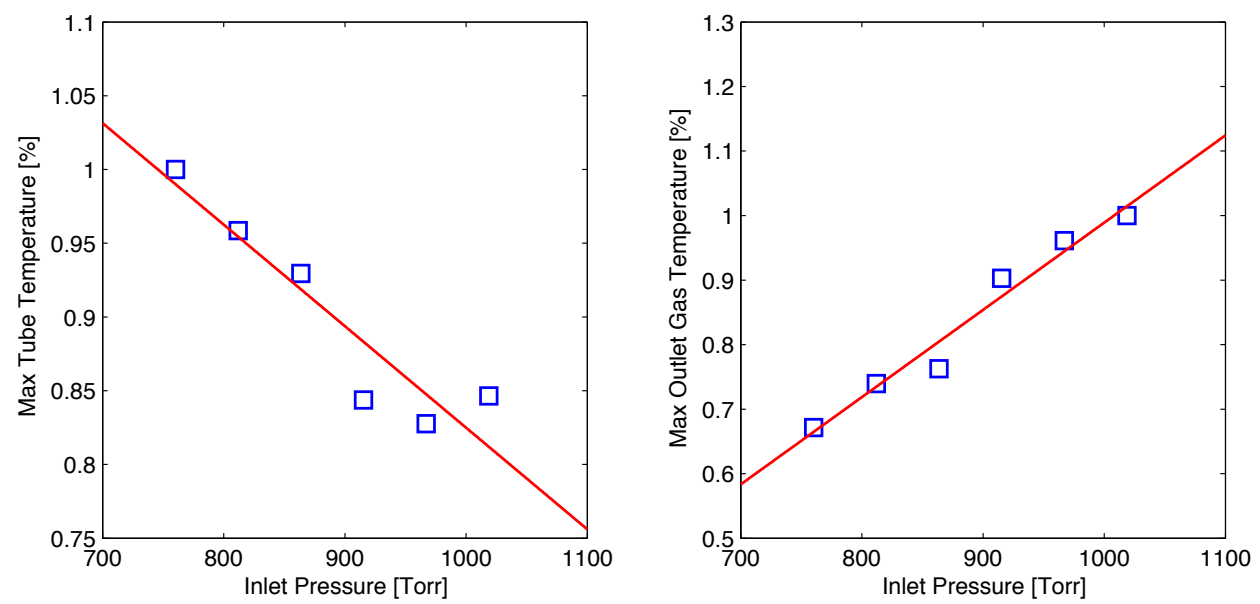

Fig. 10. Maximum temperature of quartz tube and of gas at outlet vs. gas inlet pressure.

As the carbonization system was being scaled, effluent tar buildup in the vacuum foreline, mesh filters, and vacuum pumps that led to a loss of pumping speed was noted after only several hours of processing. The loss of pumping speed meant that the chamber pressure could not be maintained for long processing runs in that configuration and the buildup of tar in the pumps led to extensive 
downtime for maintenance and rebuild. A commercial filter as shown in Fig. 11 was installed to evaluate a simple approach to removing this buildup. While the conductance through the filter had slowly reduced over time as the filter gauze fills up, the conductance was still easily high enough to maintain pumping speed even after three times the processing time required to saturate the previous mesh filter. In addition, an inspection after a significant amount of run-time revealed that far less of the effluent tars were making it past the new filter-trap, better protecting the pumps. Even with these improvements, subsequent experiments with even higher fiber throughput show that pump protection is not fully resolved.

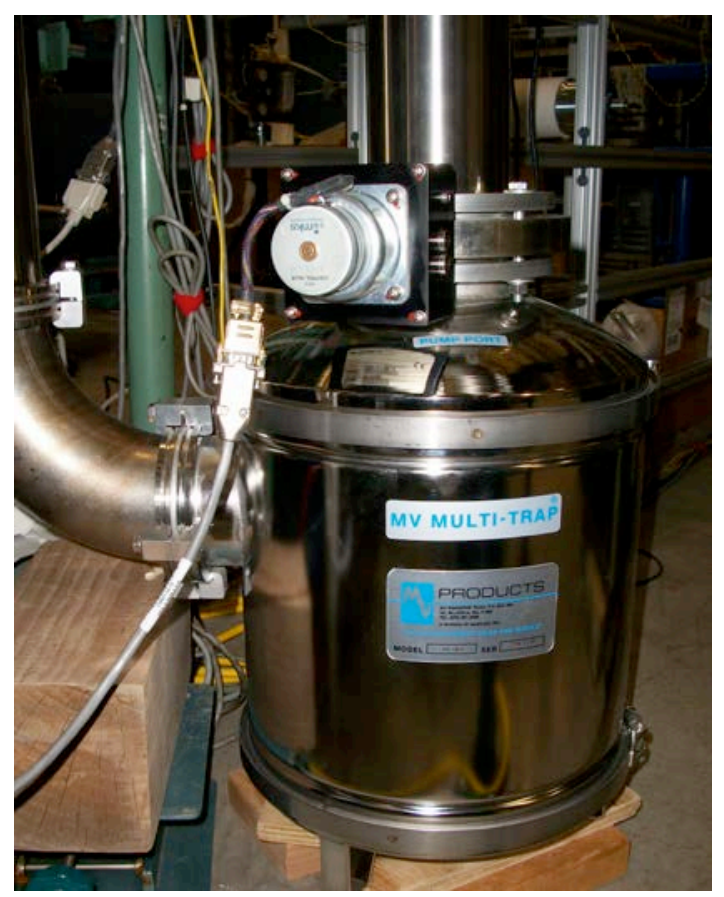

Fig. 11. Commercial filter-trap installed on the MAP vacuum foreline.

A larger diameter processing tube was installed in early stages of the scale-up. In initial runs with the larger tube, a hot spot appeared on the tube itself resulting in melting of the tube. This had been noticed before in the regions of highest plasma temperature and had been mitigated with active air cooling of the tube in these regions. To solve this new problem, three additional sources of air cooling were added to the MAP line. Comparing runs before and after the installation of the extra cooling shows no significant differences in mechanical properties. The extra cooling appeared to have solved the melting problem as was verified at the next direct inspection of the tube. Active air cooling of the tube has eliminated significant deformation of the tube and significantly increased its lifetime.

In continuing implementation and evaluation of hardware upgrades to the MAP line, the metal-toglass transition of the vacuum tube was shown to be less durable than is required for industrial use. To combat the fragility of the graded vacuum seal, an alternative mechanism using an O-ring seal was designed and installed. Replacement vacuum tubes now require just a length of tube instead of an additional pair of expensive and fragile graded seals. As an added bonus, the tube replacement time has been reduced by at least $75 \%$.

To prepare for the 5-tow experiments, the pumping sections of the MAP line were increased again in diameter to accommodate larger eyelet plates and facilitate stringing multiple tows through the line. The glass eyelets were replaced with metal eyelets. This change reduces the fragility of the system as 
well as eliminates the small level of residual rf leakage that was present at the exit of the MAP line. Given some earlier results from tow geometry experiments indicating under processing for tows positioned farther away from the rf windows, it was decided to orient the 5 tows in a vertical line centered in the vacuum tube. To accommodate the additional tows, however, the eyelets had to be placed closer together as shown in Fig. 12a.

The five-tow vertical configuration suffered from several problems stemming primarily from the feedstock. The large $50 \mathrm{~K}$ tow band obtained by the project team for the experimental work appeared to have different levels of stretch as it was wound onto the spools. (The oxidized tow being used was from a commercial manufacturer, but was provided as an experimental sample and is not representative of how that manufacturer would have produced the same tow specifically for commercial application.)

The different intra-tow stretching caused different parts of the tow to droop more or less under gravity as it was tensioned in the system (see Figure 13). Because the tows were in the vertical configuration, the less-tensioned parts of upper tows would droop down into lower tows. This would cause localized arcing and plasma formation, leading to inevitable breakage. Additionally the less-tensioned part of the lowest tow would contact the bottom of the vacuum tube leading to destruction of the tow.

To combat these problems, the tows were repositioned to a horizontal linear orientation as shown in Figure 12b. It was found after testing that the innermost and outermost tows do not touch the vacuum tube and adjacent tows do not appear to interact in the same way as in the vertical configuration.

To meet an 8 hour intermediate level run requirement, three tows were placed horizontally as in Fig. 12(b). There was a concern that the outer tows farther away from the rf input might be under processed, but this position allowed for expediently evaluating hardware durability and vacuum feedthrough issues more critical to long-term success. A continuous $81 / 2$ hour run was successfully completed with the three tows. There were no problems at all with the vacuum tube or the pumping systems. Inline linear resistance measurements during the run indicate that as expected, the outermost tow is less processed that the other two. Average mechanical properties for these tows did meet project targets.
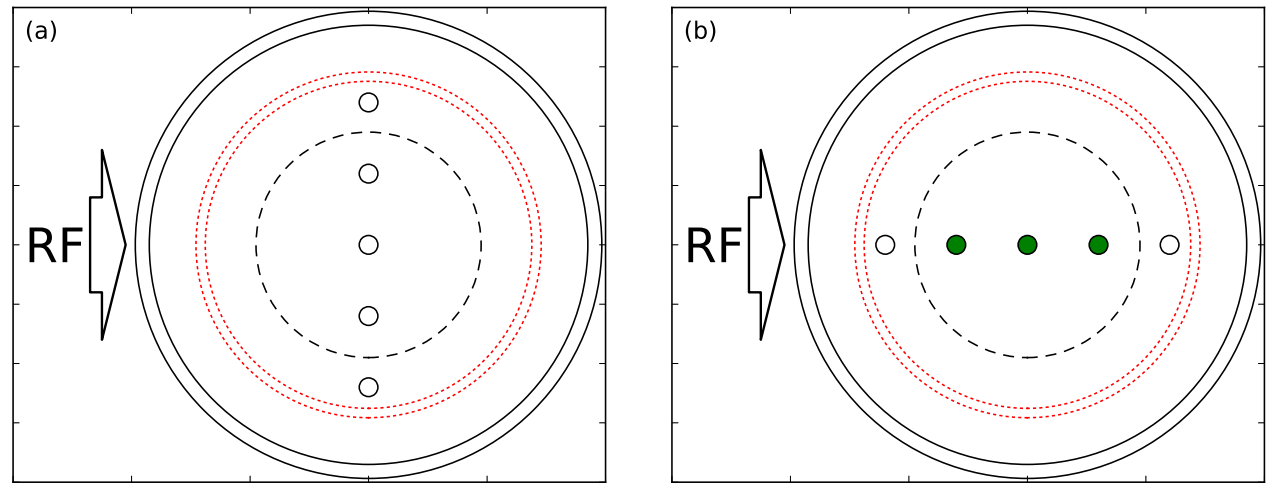

Fig. 12. Geometry of the tows within the vacuum tube. The earlier configuration (a) had the tows oriented vertically while most recently this was changed to a horizontal orientation (b). The red dotted circles are the extent of the old, smaller tube used for 3-tow experiments in 2011. The dashed circle prescribes the radius of the outermost tows prior to the 5-tow experiments. 


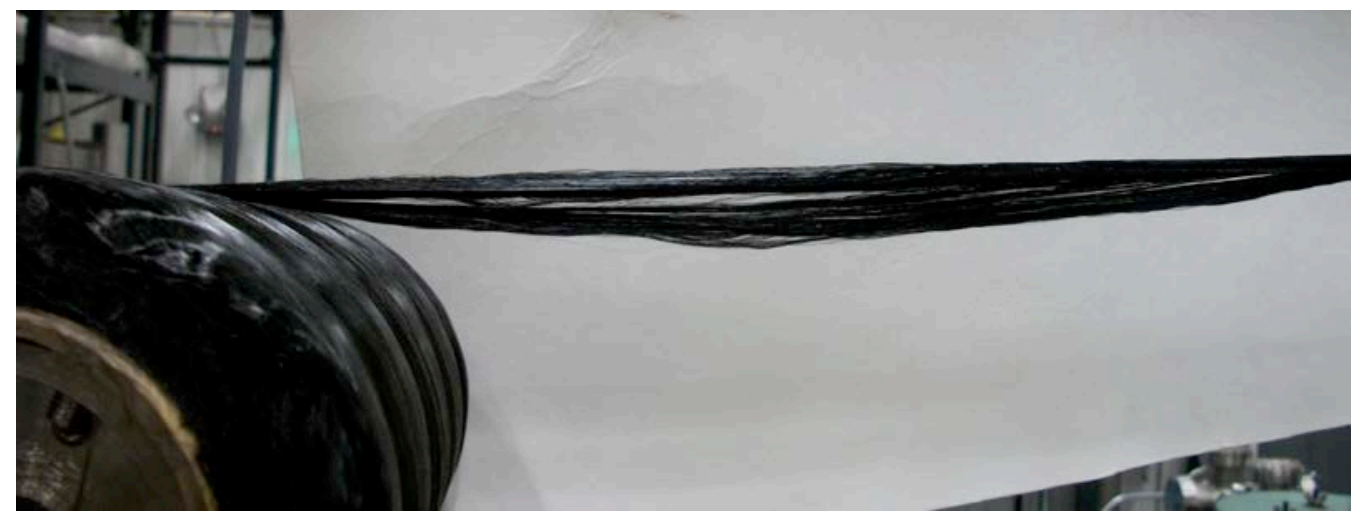

Fig. 13. Intra-tow tension differences in oxidized PAN feedstock. Part of the tow is under very little tension.

Initial work with five tows in the applicator indicated that the horizontal tows are not processed evenly, with the tow farthest from the coupled rf inadequately processed as shown in Fig. 14. The solution to this issue was to rotate the rf applicator itself so that the tows are still horizontal to avoid gravity effects, but with all the tows a similar distance from the rf source. This orientation is shown in Fig. 14c. This rotation was completed and the updated configuration is shown in Fig. 15.
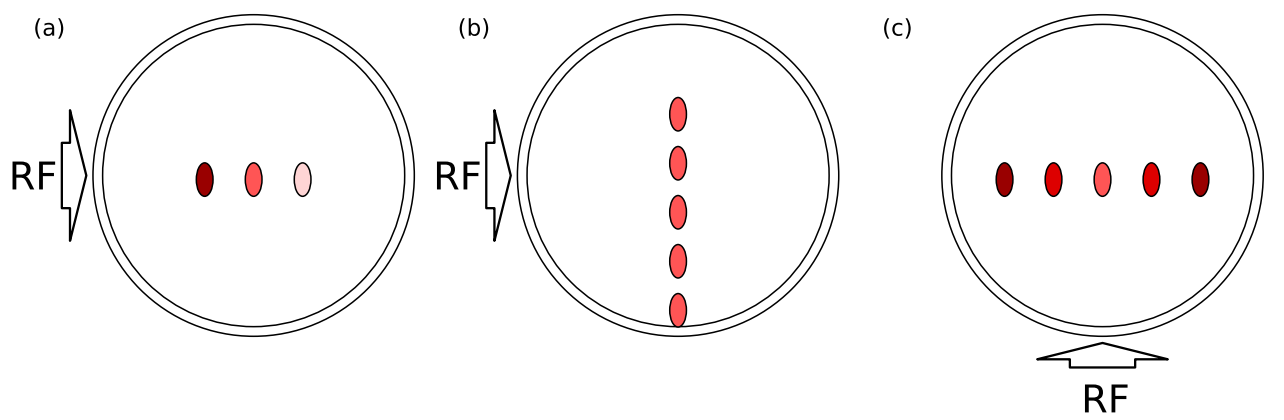

Fig. 14. Tow orientations within MAP applicator. (a) Orientation in pre-2012 multi-tow experiments. Heating is very uneven. (b) Attempted tow orientation in 3Q2012 campaign. (c) Tow orientation after applicator rotation for 5-tow experiment in early FY 13.
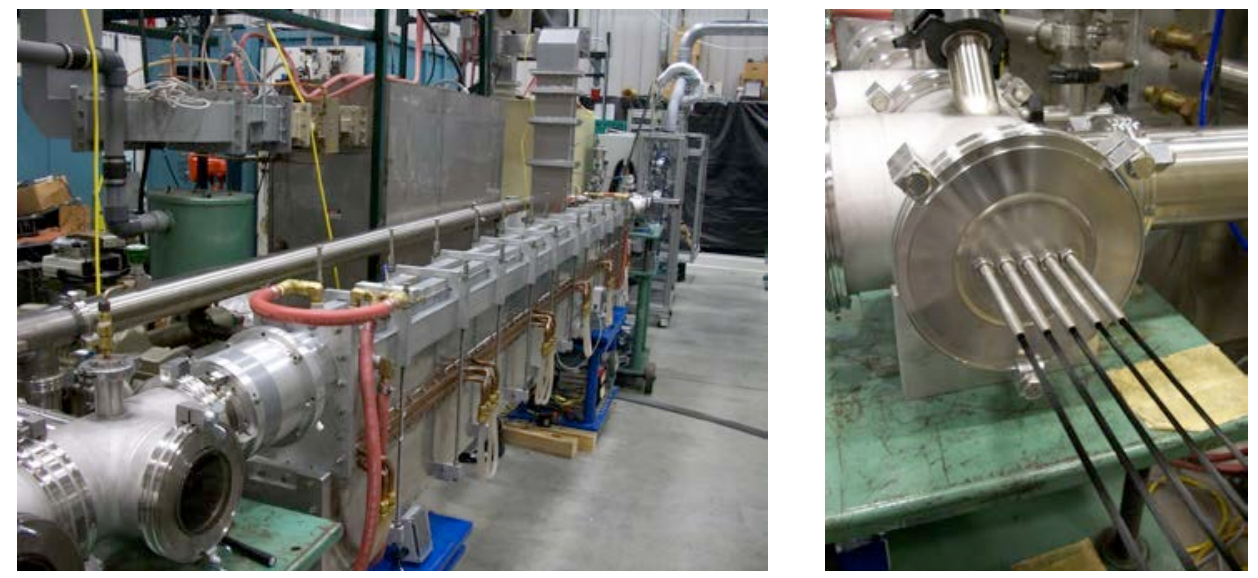

Fig. 15 . (a) The MAP line after rotating the applicator. Microwave coupling is now from below. (b) The MAP line exit feed-throughs are shown fully strung with five tows in the horizontal configuration. 
With the rotated MAP applicator and five horizontal tows, the plasma was stable over a larger operating space than had seen under 3-tow operation. Reflected power was on average several percent lower than in 1 or 3-tow operations as well, which raises the efficiency of the process. However, preliminary experiments found that tow-to-tow processing is not as uniform as had been hoped. The outer tows seem to be processed more than the inner tows, but the variance is not as drastic as was seen in the 3-tow experiments with the rf coupling from the side. Another internal milestone was completed with a two hour MAP run with five tows. Several parameters are shown in Fig. 16. For the most part this was a smooth run, with the exception that at about 17 minutes into the run, the microwave generator tripped off. The cause is unknown, but is not believed to relate to the MAP process itself. After resetting, the team was able to achieve the prior operating conditions about two minutes later. (Actually it is believed that the ability to stop, start, and change parameters rapidly as necessary is one of the inherent advantages of the MAP approach over conventional processing.) After the reset, the run continued uninterrupted for another two hours and six minutes before shutting down normally.
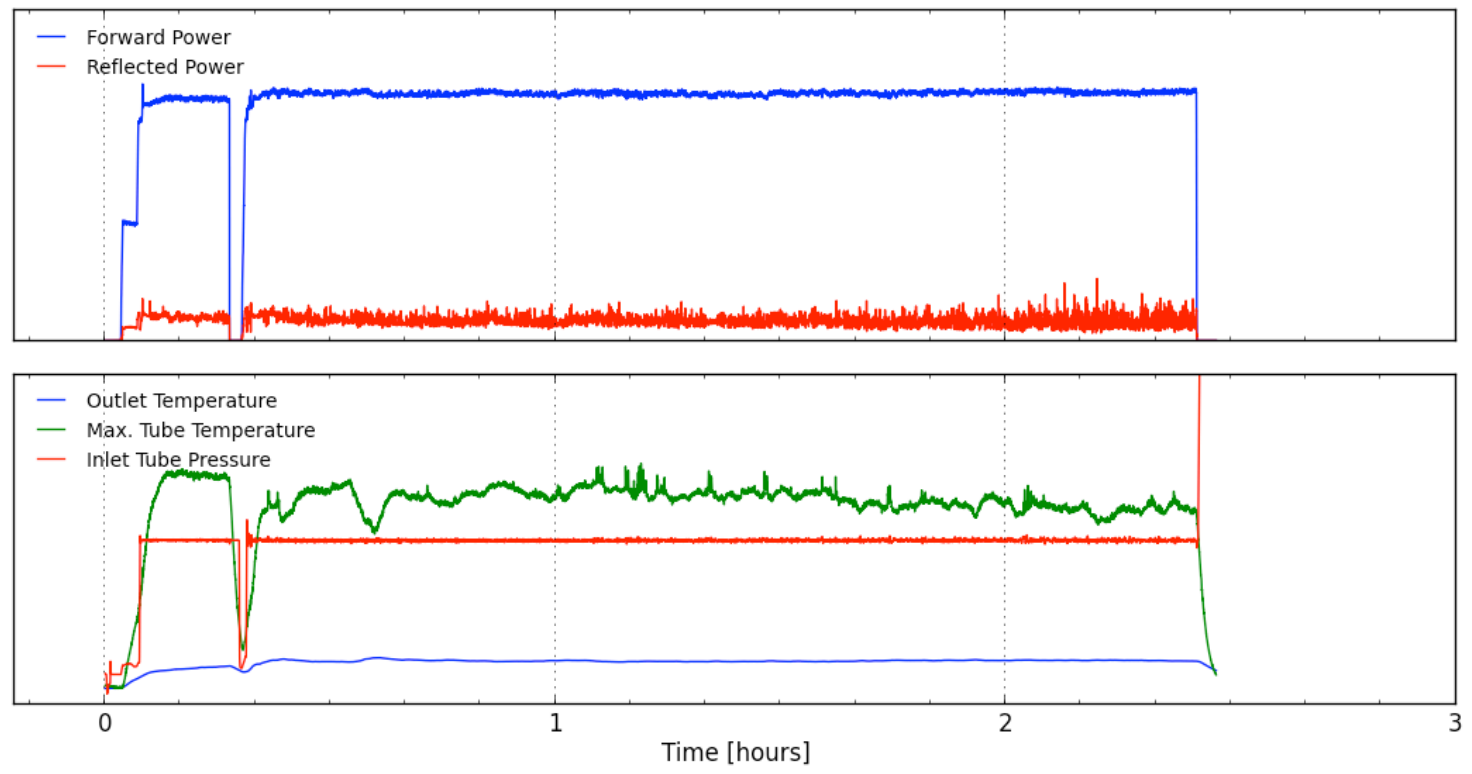

Fig. 16. Time trace of 5-tow, $2+$ hour milestone run. Shown are the forward and reflected power, the nominal maximum vacuum tube temperature, the outlet gas temperature, and the tube gas pressure. At $\mathrm{t}=17$ minutes, the microwave generator tripped.

Since implementing the larger processing chamber tube and transitioning to 5-tow operation, operations continued to improve allowing focus on issues such as factors influencing capability of the system to produce larger quantities of fiber at higher rates and capability to produce more consistent materials. Fig. 17 gives a compilation of results in running with five tows including indication that mechanical properties are approaching targets even at 500\% increased throughput over previous three tow operation. In terms of variability, the mechanical properties of the interior tows were significantly lower than for the exterior tows in the initial work with five tows. A run with similar operating parameters, but at $50 \%$ more speed demonstrated some degradation of properties, but the property levels were in ranges comparable with earlier experiments where relatively small modifications to processing parameters allowed achievement of targets. Indeed, by altering the operating parameters at the lower speed, the team was able to increase the tensile strength and 
modulus to a point where the target specification for all tows as shown by the cyan points in the figure was met.

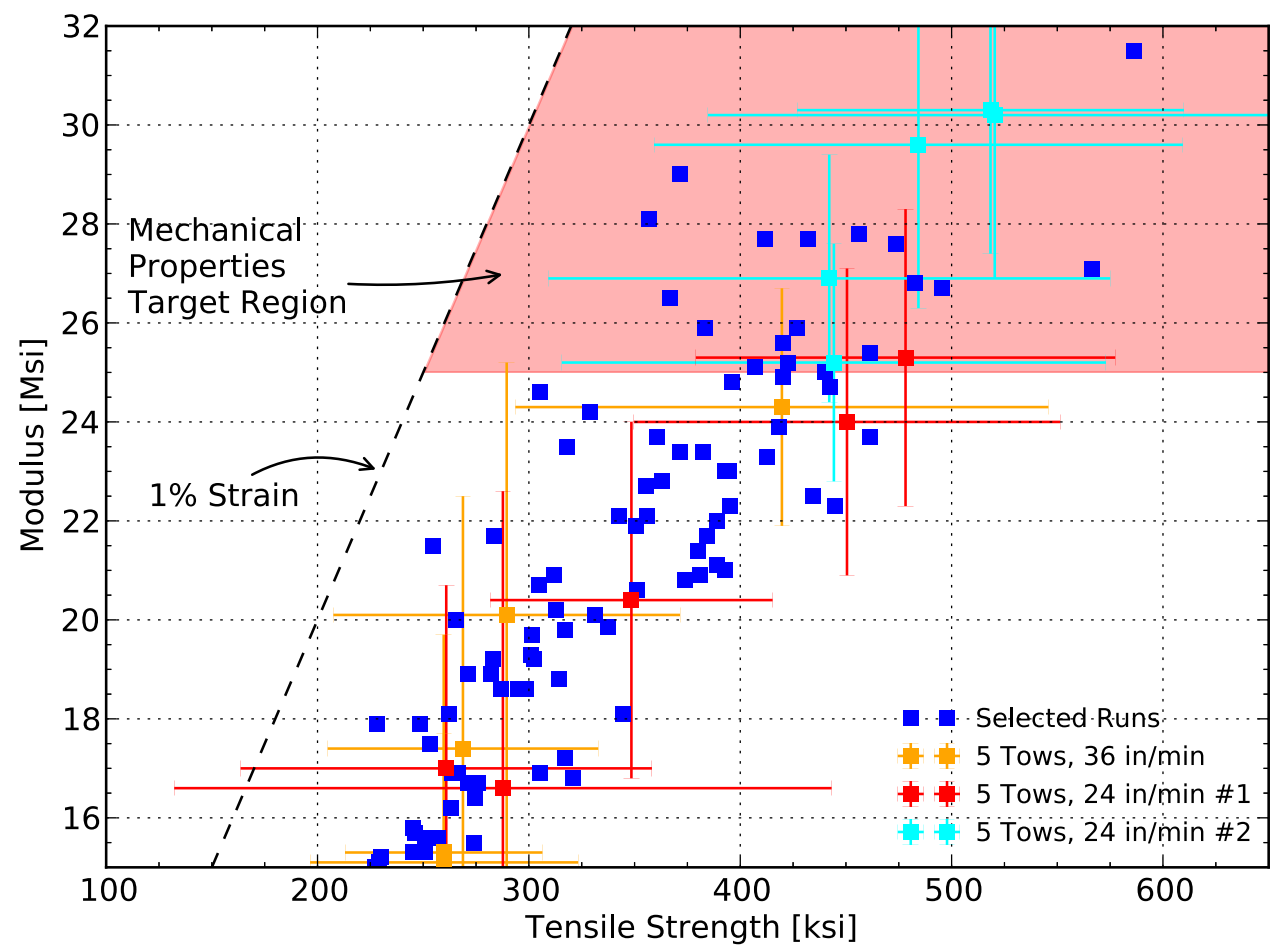

Fig. 17. Mechanical properties from selected MAP runs. The orange, red and cyan points are from three 5-tow runs. Fiber properties in the shaded region meet or exceed programmatic targets. Large variance is due to single-filament mechanical testing.

Multiple experiments have been performed to determine routes for improving tow-to-tow variability. Several approaches were identified and upgrades to the MAP system were completed to demonstrate effectiveness of the identified approaches including changing the tow configuration in the processing chamber and modifying the microwave energy introduction and management approach as described below.

Previous five-tow experiments have been able to exceed the programmatic mechanical property targets; however, it was found that the tows suffer from a tow-to-tow variability with the central tow just meeting the modulus target while the outer tows exceeded the modulus target by over $20 \%$.

Guided by energy deposition modeling and observation that some wall heating might be affecting the level of processing, tow configuration changes were implemented. Fig. 18 shows the results of a previous experiment on the left and an experiment with the modified "smiley-face" configuration on the right, with both experiments using SGL Panox 50K tows at approximately the same operating parameters including a tow movement speed of $24 \mathrm{in} / \mathrm{min}$. As shown in the Fig. 18, the resulting data shows average properties improved overall in the new configuration, but the configuration changes resulted in reversed trends: the mechanical properties have now switched positions with the middle tows experiencing greater conversion and the outer tows processed less thoroughly. Mechanical properties were not measured along the fiber length, but density and resistivity measurements along the fiber length indicate consistent uniformity. This result indicates that shifting the tows radially can indeed influence the inter-tow properties and we expect that making the configuration a little less "smiley" should even up the mechanical properties even more. From the data differences, it is surmised that any wall heating is a second order effect. 

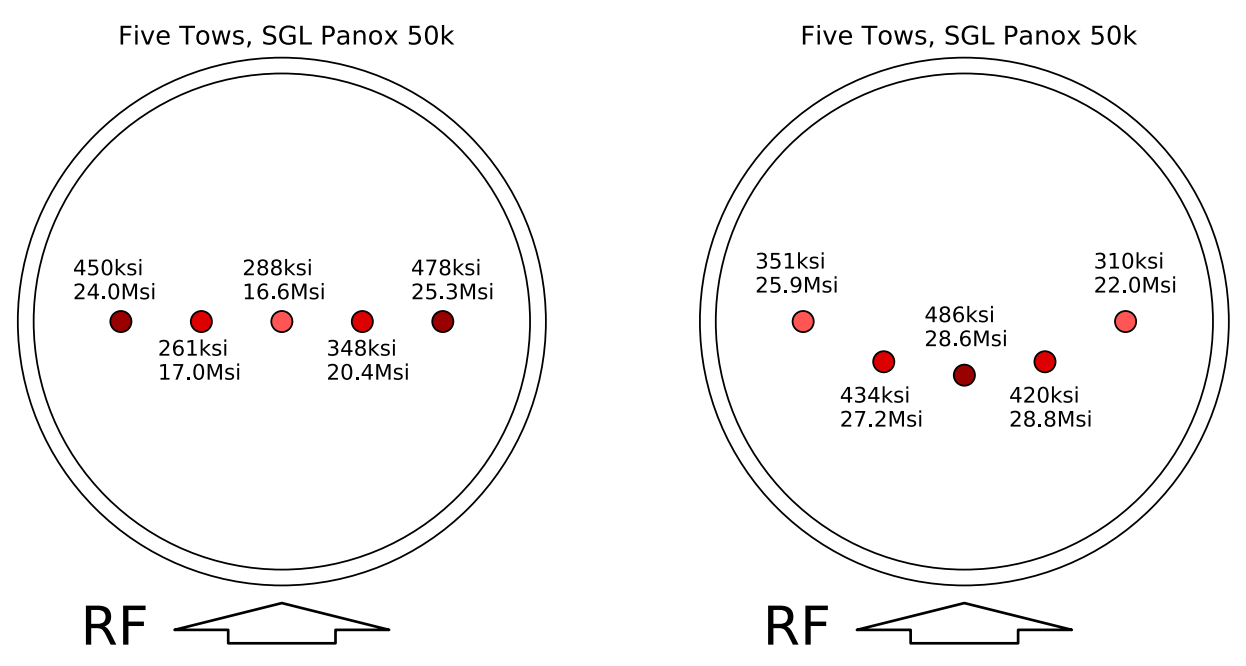

Fig. 18. Mechanical properties and relative positions of a single 5-tow MAP run for the horizontal configuration (left) and the "smiley-face" configuration (right).

Perhaps having the greatest potential impact in process management, among the modified approaches implemented for evaluation during the fourth quarter of FY2013 was an alternative technique based on existing microwave technology and relatively standard hardware that exploits modular microwaveplasma cavities. To validate the approach prior to reconfiguring the entire processing system, a single unit was tested in the MAP system initially.

Plasma stability and reproducibility has long been a focus in moving the MAP line to a dependable industrial process. The number of zones and relative power and location of the multiple plasma zones in the side-walled coupled waveguide MAP applicator are highly interdependent. System stability is a function of the input power and pressure as well as the location and presence of the carbon fiber in the applicator. If one could uncouple the plasma zones from each other, then the heating profile can be more easily tailored and also the plasma can be made more stable under a variety of operating conditions. Fig. 19 shows a proposed multi-cavity applicator method that utilizes commercially demonstrated hardware to replace the side-wall coupled design for the MAP. Each cavity independent energy deposition control and can be matched to provide the desired cavity-to-cavity heating profile. 


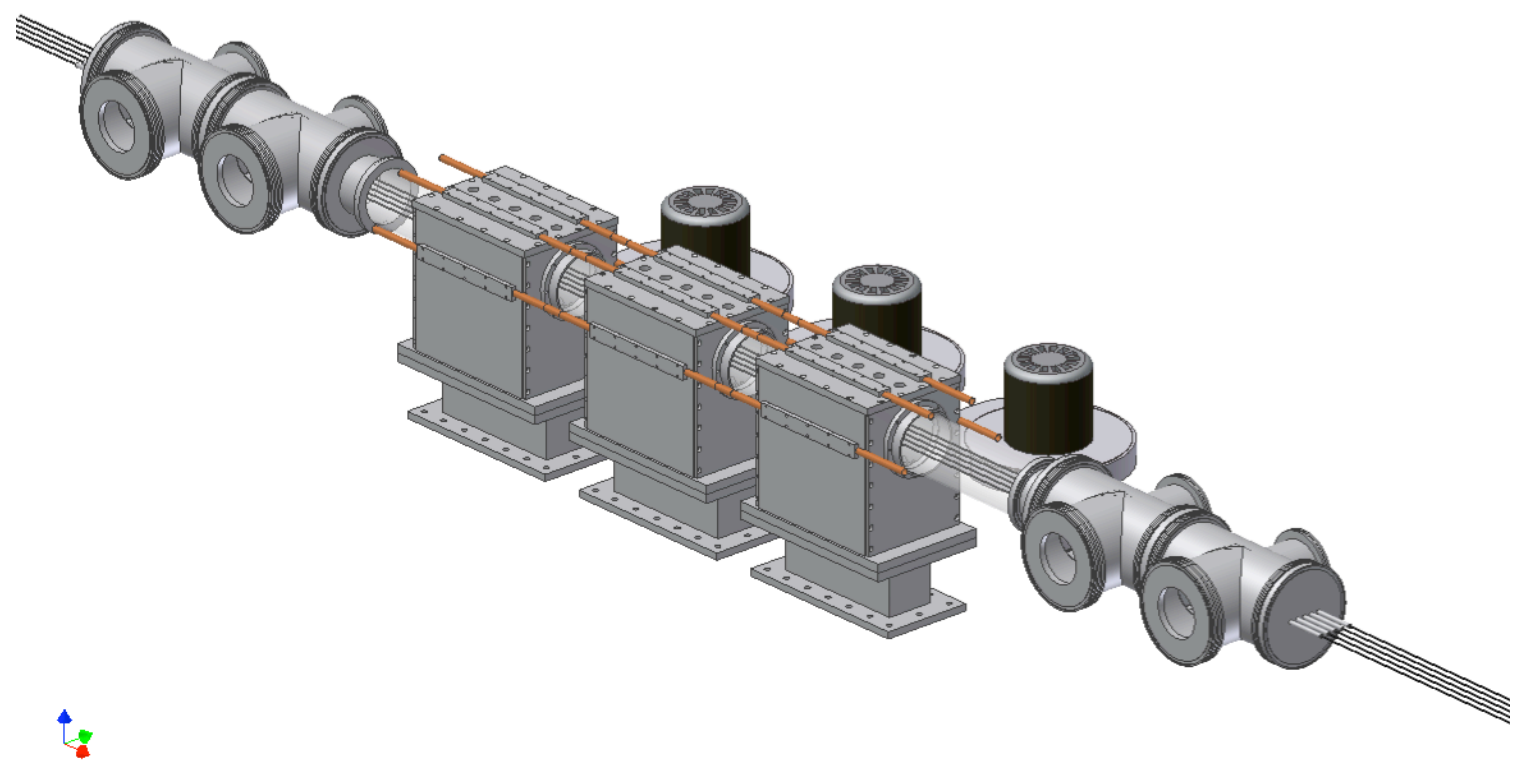

Fig. 19. Proposed multi-cavity MAP applicator. Each cavity should be nominally uncoupled from the others, unlike the plasma zones in the side-wall coupled applicator.

In order to demonstrate feasibility of this concept, a single unit cavity was obtained, installed and tested. Figures 20 and 21 show the new single unit applicator, referred to as MAPv4.1. (In order to simplify discussion, versions of the MAP system with the new energy deposition approach are designated as MAPv4.X or later to distinguish from versions based on earlier technology designated as MAPv3.X or below.) Medium power rf tests demonstrated excellent directivity and plasma tests at low power provided excellent coupling and an ability to maintain what is expected to be attractive processing parameters even at low input power. Fig. 21 shows a plasma in the cavity running a single $50 \mathrm{k}$ tow. Mechanical testing of the $50 \mathrm{k}$ tow running at $24 \mathrm{in} / \mathrm{min}$ yielded $376 \mathrm{ksi}$ tensile strength and 23 Msi modulus - slightly lower than our programmatic targets. However, this is extremely encouraging as the results were achieved with only a single cavity with un-optimized processing parameters.

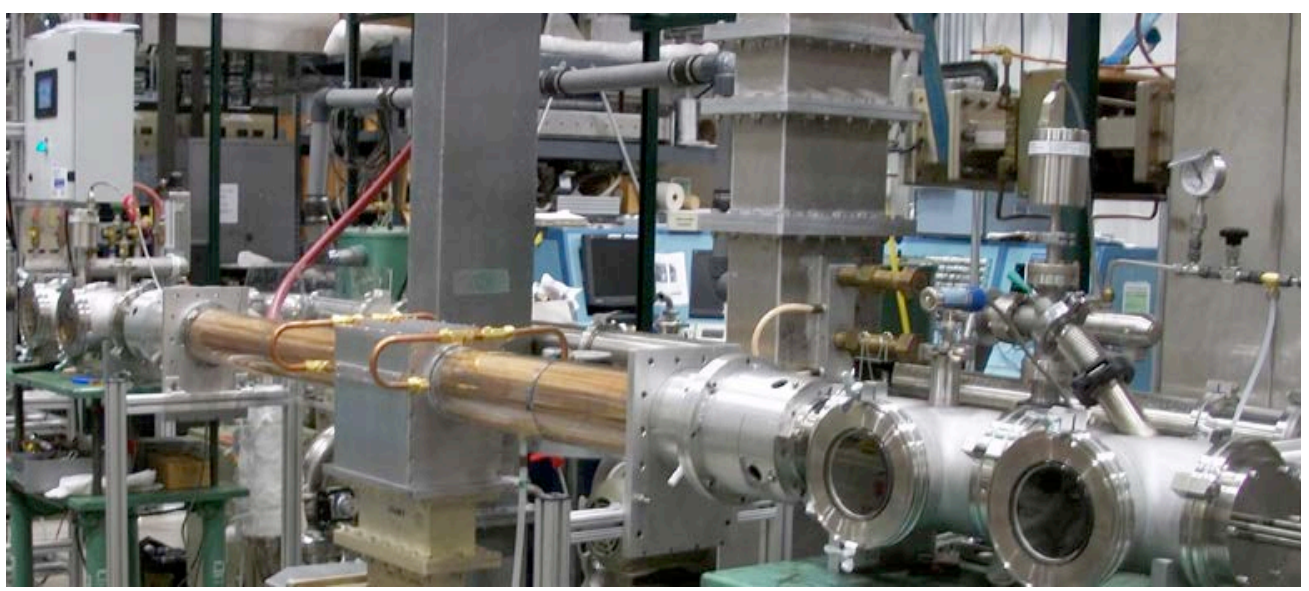

Fig. 20. The new single-cavity MAP applicator. 


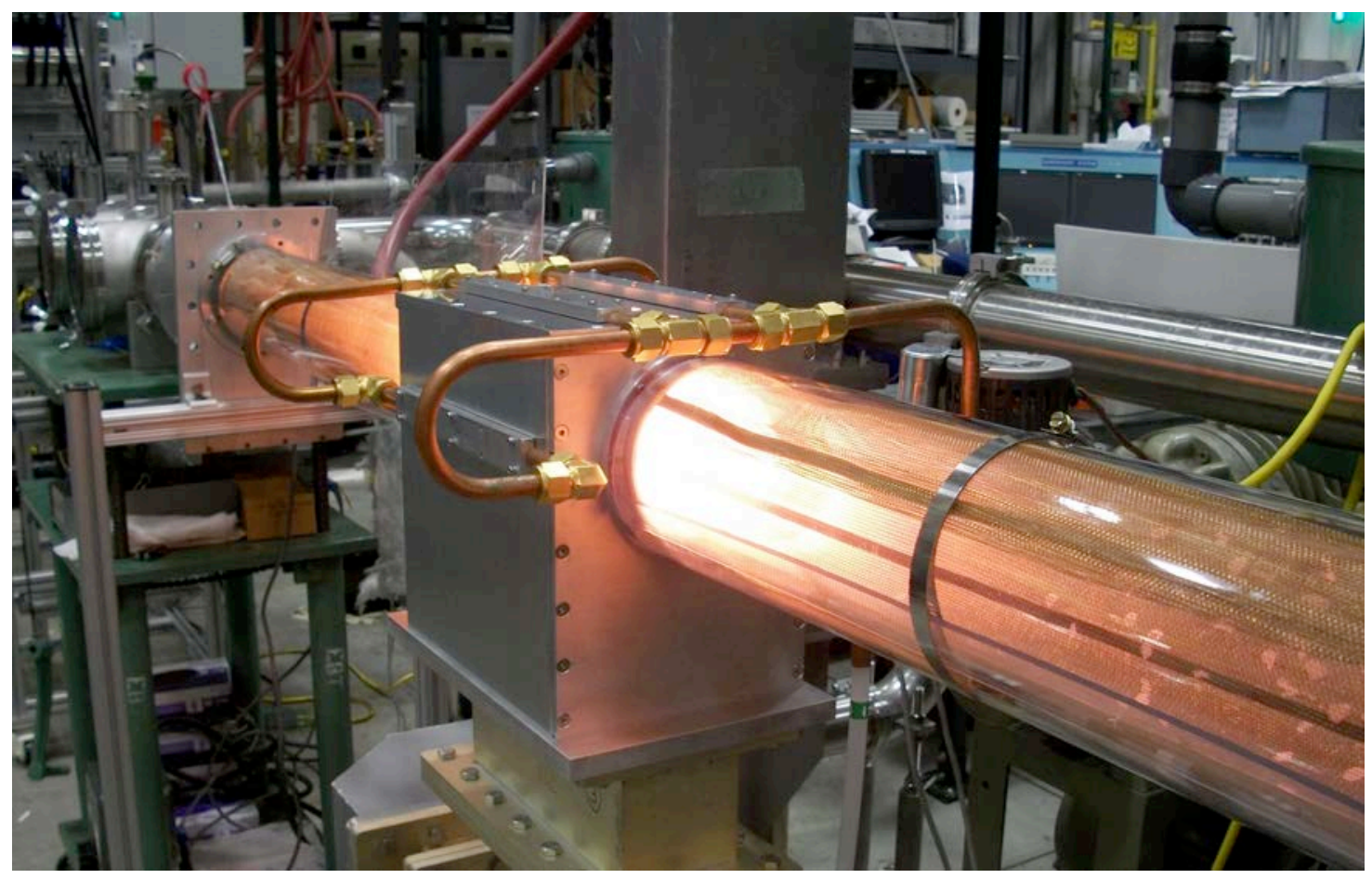

Fig. 21. A plasma test with a single tow in the v4 MAP applicator.

With these results the team decided to go forward and add two or three additional cavities to the applicator for full carbonization capability. In the last quarter of the project, the MAP system was modified for experiments with a tow cavity system referred to as MAPv4.2 and again upgraded for a three cavity system referred to as MAPv4.3. The two-cavity applicator (MAPv4.2) allows for singletow operation at or above our programmatic targets with speeds up to $5 \mathrm{ft} / \mathrm{min}$. For proper distribution of power into the second cavity, input power was increased by $15-40 \%$ relative to the MAPv4.1 experiments (but still almost 50\% less microwave power than for the side-wall slot coupled applicator of MAPv3).

Three 50k tow operation with the MAPv4.2 was attempted with some success, but at the higher speeds, the center tow of the three was being processed much less than the outer tows. The line was upgraded again to three cavities (MAPv4.3). The applicator is shown in Fig.22. For the experiments done in this quarter, the input power was only increased by $\sim 5 \%$. Because of the problems we had processing five tows in MAPv4.1, the tow geometry configuration was changed to a circular pattern as shown in Fig. 23. For five tows, the top position was not used. With this configuration, all five tows can survive full power, but the bottom tow consistently shows less processing than the other four overall operating parameters. With this data, it was decided to reduce the total number of tows to four. This still allows a CFTF scale machine with three MAP modules $(4 \times 3=12 \times 50 \mathrm{k}$ tows $24 \mathrm{x}$ $24 \mathrm{k}$ tows). Processing with four tows gives much better inter-tow consistency with a standard deviation of only $\sim 50 \mathrm{ksi}$ tensile strength and $\sim 0.75 \mathrm{Msi}$ modulus at $5 \mathrm{ft} / \mathrm{min}$. 


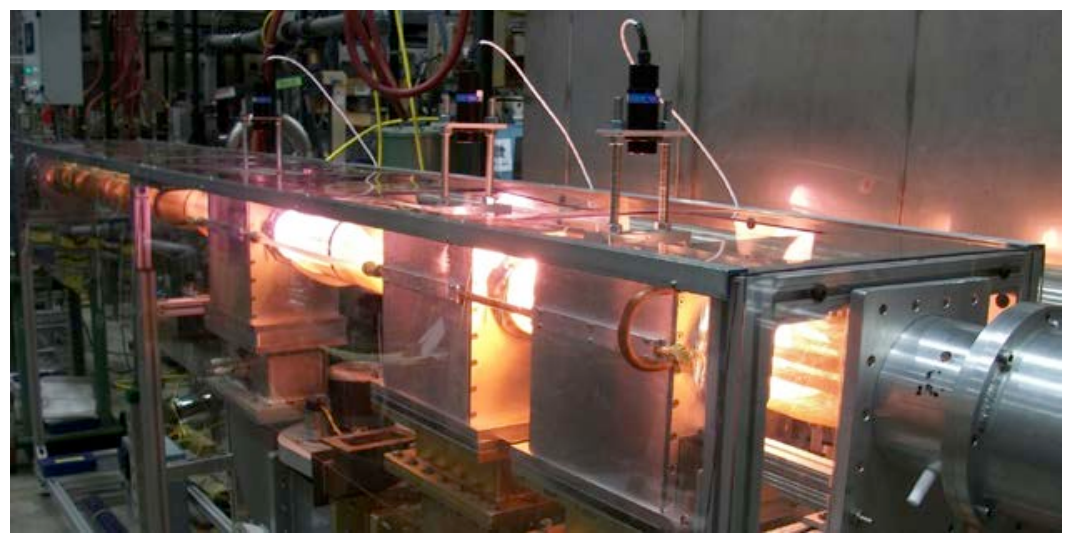

Fig. 22. The triple-cavity MAP applicator shown here with rf and UV shielding.

Additionally, five 50k tows were combined into a single $250 \mathrm{k}$ tow and processed through the MAPv4.2. Fig. 23 shows the single eyelet for the $250 \mathrm{k}$ tow. The average mechanical properties of the tow exceeded our target at $2 \mathrm{ft} / \mathrm{min}$; however, there was some intra-tow variability: some parts of the tow are not being processed as much as others. Doubling the speed to $4 \mathrm{ft} / \mathrm{min}$ decreases the tensile strength by $50 \%$ and the modulus by $30 \%$. Experiments with the $250 \mathrm{k}$ tow on the triple-cavity MAPv4.3 initiated by the end of the quarter were not able to obtain mechanical properties. Fig. 24 shows the results of these recent notable runs with other historical data.

Work also continued on handling the MAP exhaust effluent. Our previous MV Products Multi-Trap filter was replaced with a custom ceramic filter shown in Fig. 25. This filter is fully bake-able to regenerate the filter. Initial performance of the new filter is promising. Despite being less porous than the Multi-Trap filters, the lower conductance of the filter is not an impediment to vacuum pumping the system. Inspection of the upstream vacuum foreline after a high throughput run shows much better protection of the vacuum pump with the new filter.
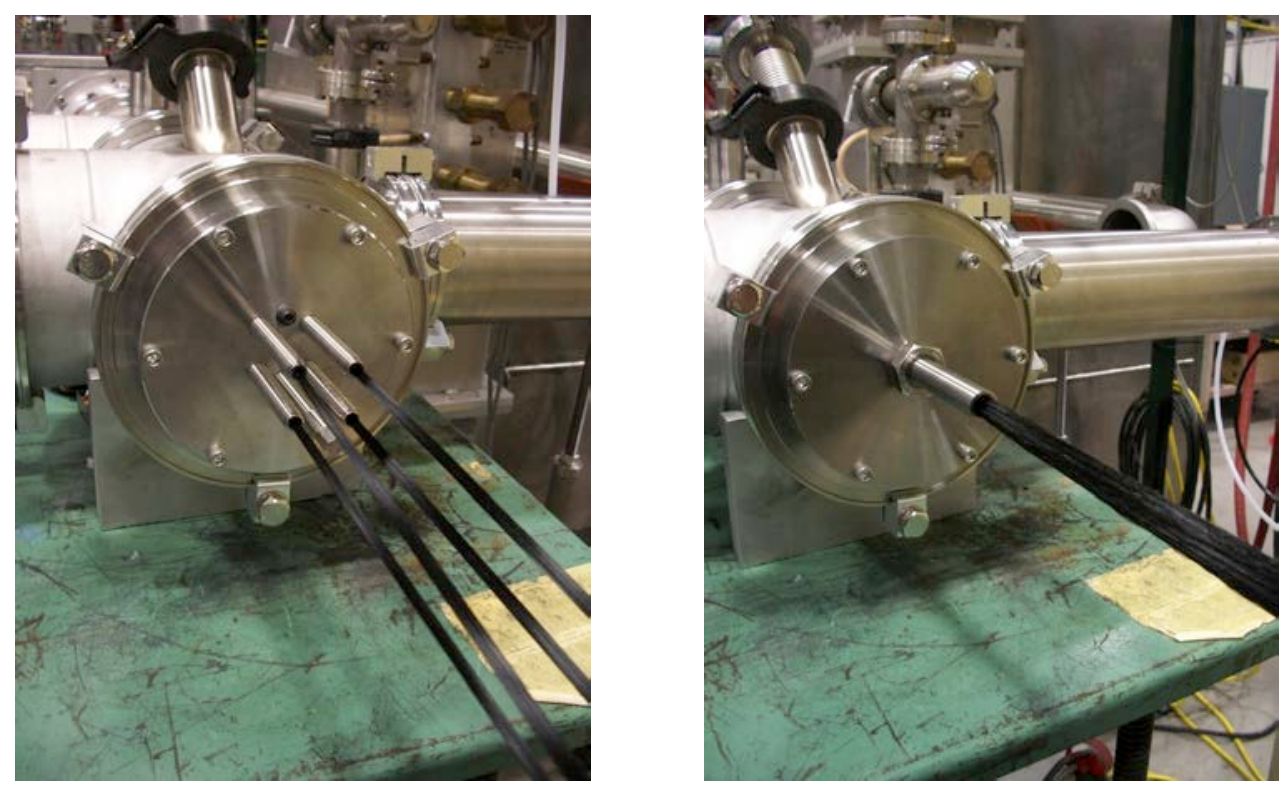

Fig. 23. The tow configurations for $450 \mathrm{k}$ tows and a single large $250 \mathrm{k}$ tow. 


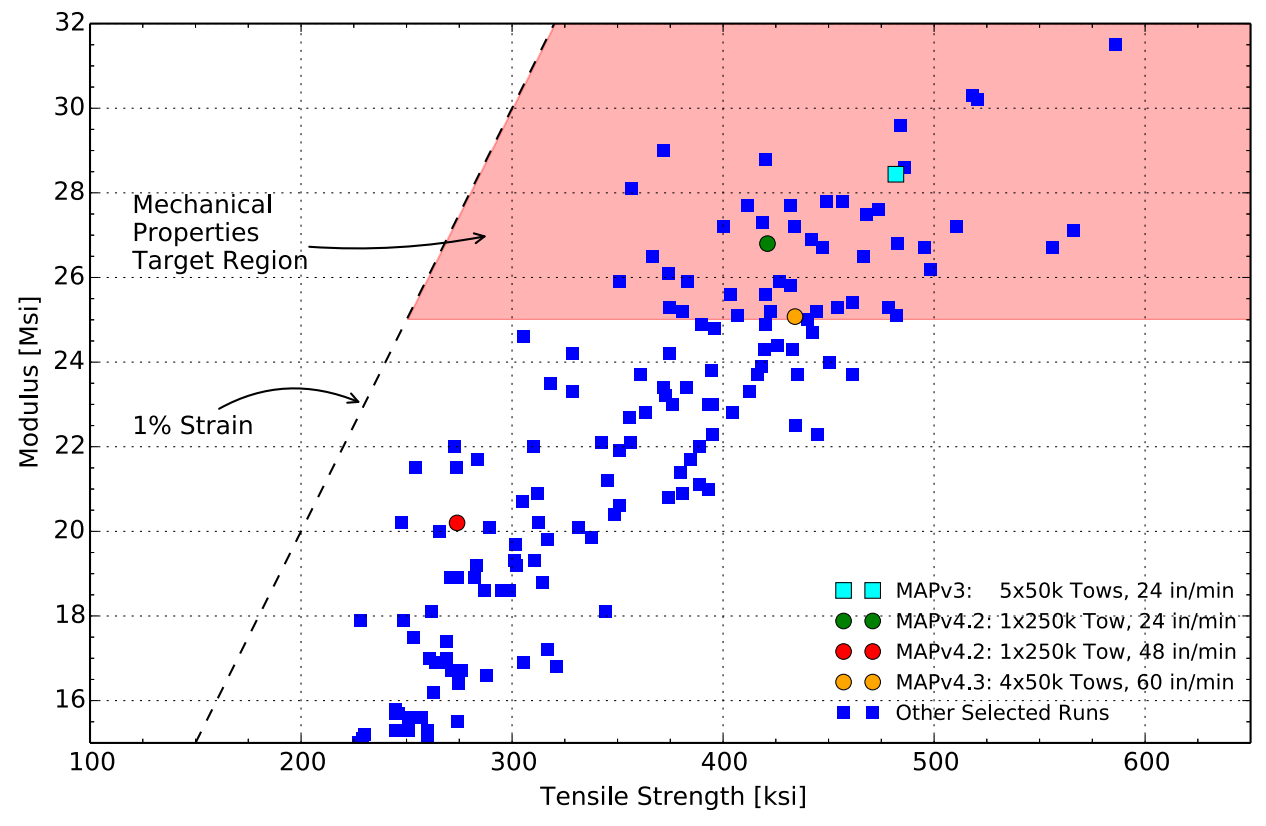

Fig. 24. Mechanical properties of MAP-processed carbon fiber for selected runs. MAPv4.2 corresponds to a dual-cavity system; MAPv4.3 is the triple-cavity system. The properties for multi-tow runs are averaged over all tows.

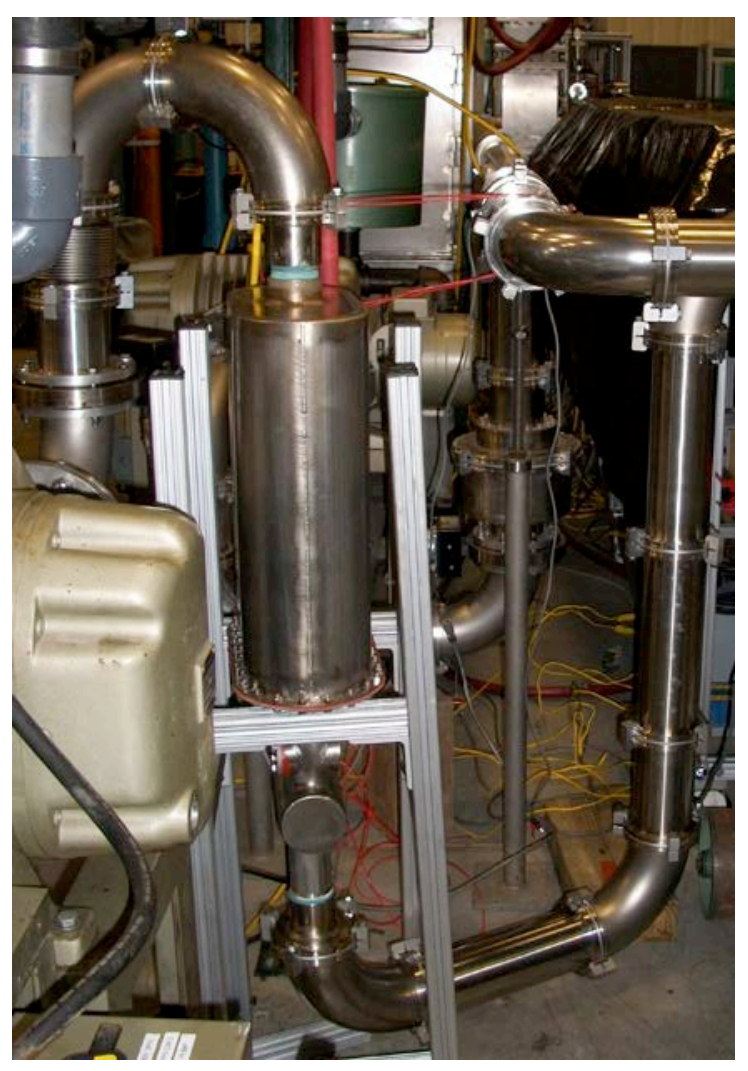

Fig. 25. New ceramic filter system in line with primary vacuum line. 


\subsection{MAP ENERGY BALANCE}

To effectively take MAP processing to an industrial scale, we must have data regarding energy usage as well as the various precursors and products. A schematic of the MAP system is shown in Fig. 26. In addition to the oxidized fiber precursor, inputs include a cover gas to maintain an inert atmosphere, water and air for cooling and electrical power. The water is circulated through a heat exchanger and is in a closed loop. For the energy balance calculation, the power consumed by the heat exchanger is not computed. Instead the amount of power removed by the water is measured. This value will set the requirements for a future dedicated cooling system for an industrial-scale MAP. The other outputs are cooling air, the carbonized fiber, and residue and exhaust gases.

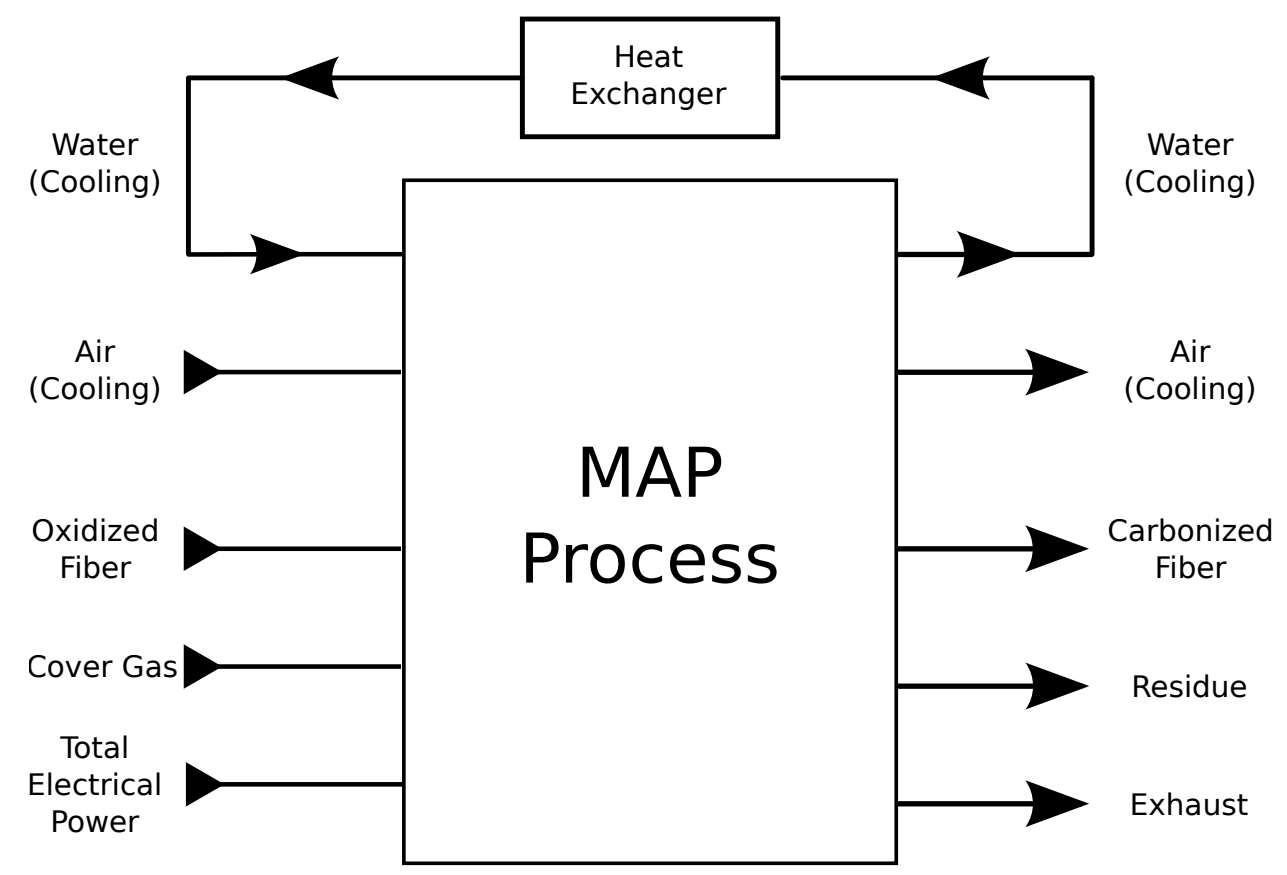

Fig. 26. Energy and mass inputs and outputs for the MAP process.

The required electrical power is used for microwave generation, cooling fans, vacuum pumping, and diagnostics. For the baseline energy balance, the power for the microwave generator comprises about $65 \%$ of the total electrical input. The exhaust consists of the cover gas output as well as any gaseous byproducts of the conversion process. The residue consists of any other non-gaseous vapors (the tars mentioned previously) of the effluent. Data from conventional processing shows that this residue is approximately $20 \%$ by mass of the oxidized precursor fiber.

It remains to be seen exactly how processing efficiency scales. Multi-tow experiments show that doubling the throughput of the MAP line required only $\sim 15 \%$ more RF input power to maintain equivalent mechanical properties. This figure corresponds to a $\sim 6 \%$ increase in the overall electrical power needs of the current MAP system. As tows are added to a single applicator (read module), we should expect favorable $\mathrm{kW} / \mathrm{lb}$. scaling. The energy balance calculation will continue to be refined as parameters are tuned to optimize performance and the system is scaled up.

\subsection{ALTERNATIVE PROCESS INVESTIGATIONS}

In parallel to the work described above, the team also evaluated the feasibility of some atmospheric pressure alternatives to significantly reduce or eliminate the sealing issue altogether. A review of the 
current literature indicated that the use of a plasma torch or set of plasma torches might be a promising avenue of research. As with the baseline MAP process, any such solution must be evaluated with an eye toward the possibility or difficulty of industrial scale-up. Previous attempts to perform carbonization/graphitization at atmospheric pressure did not prove fruitful. There were potentially several reasons for this lack of positive results, the most pressing being that the torch power was limited to greater than $1 \mathrm{~kW}$ in order for the torch to operate in a stable manner. More recently published work with both the earlier inductively coupled plasma (ICP) type torch and the more recent microwave waveguide type torches has allowed the power levels to be reduced by more than an order of magnitude while still maintaining stable operation. An array of such low power torches might be necessary for scale-up to multiple tows if single tow operation and control could be demonstrated.

A low power atmospheric pressure plasma torch based on the "surfaguide" concept was constructed as shown in Fig. 27. Initial tests have demonstrated that the torch can be operated with argon with low as $80 \mathrm{~W}$ of input power. Some axial temperature profiles are shown in Fig. 28. A plasma was successfully formed with nitrogen as the fill gas; however, the minimum power obtained was $100 \mathrm{~W}$ before the plasma self-terminated. Additionally, certain plasma instabilities with nitrogen were observed that might be due to the torch design.

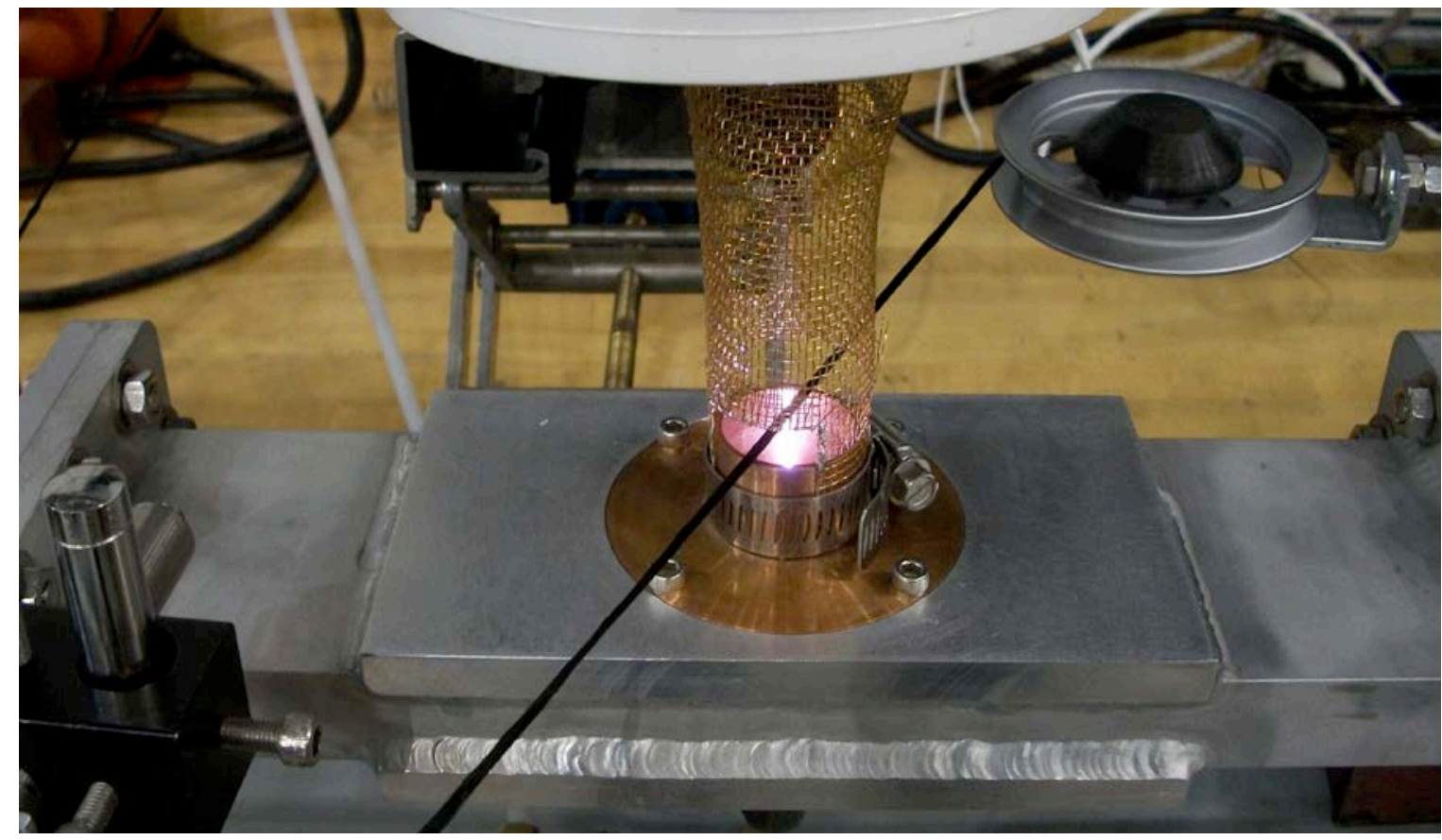

Fig. 27. Atmospheric pressure plasma torch operating with oxidized tow.

A static test of carbonization with an oxidized tow $\sim 20 \mathrm{~mm}$ above the torch nozzle at $200 \mathrm{~W}$ showed no degradation. Slowly moving the tow through the flame quickly frayed and broke the tow, an indication that too much oxygen was present in the vicinity of the flame. Subsequent tests with a partial nitrogen blanket did not prevent the destruction of the tow. The experiment was modified to provide for a completely inert atmosphere near the plasma flame. To do so, a "bell jar" was designed and fabricated to keep the torch under an inert atmosphere. The improved design is shown in Fig. 29. With a nitrogen blanket at $<30 \mathrm{ppm} \mathrm{O}_{2}$, a preliminary experiment with a fully carbonized tow was performed. At modest line speeds (less than $20 \mathrm{in} / \mathrm{min}$ ), the temperature of the tow was measured to be $\sim 700^{\circ} \mathrm{C}$, sufficient for low-temperature carbonization. This was accomplished with minimal 
fraying and $<100 \mathrm{~W}$ of input power.

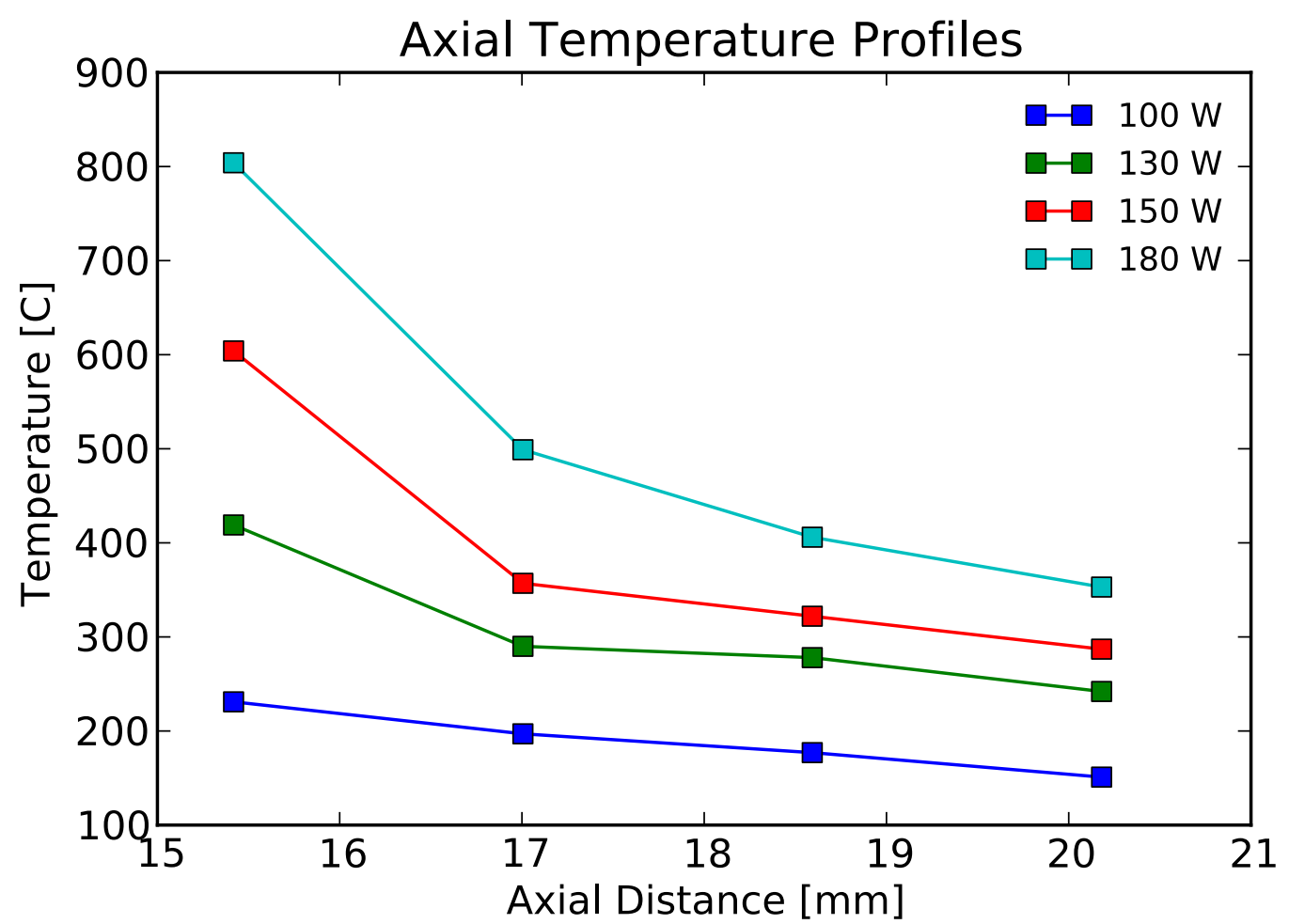

Fig. 28. Temperature profiles with respect to the axial distance from the plasma torch nozzle.

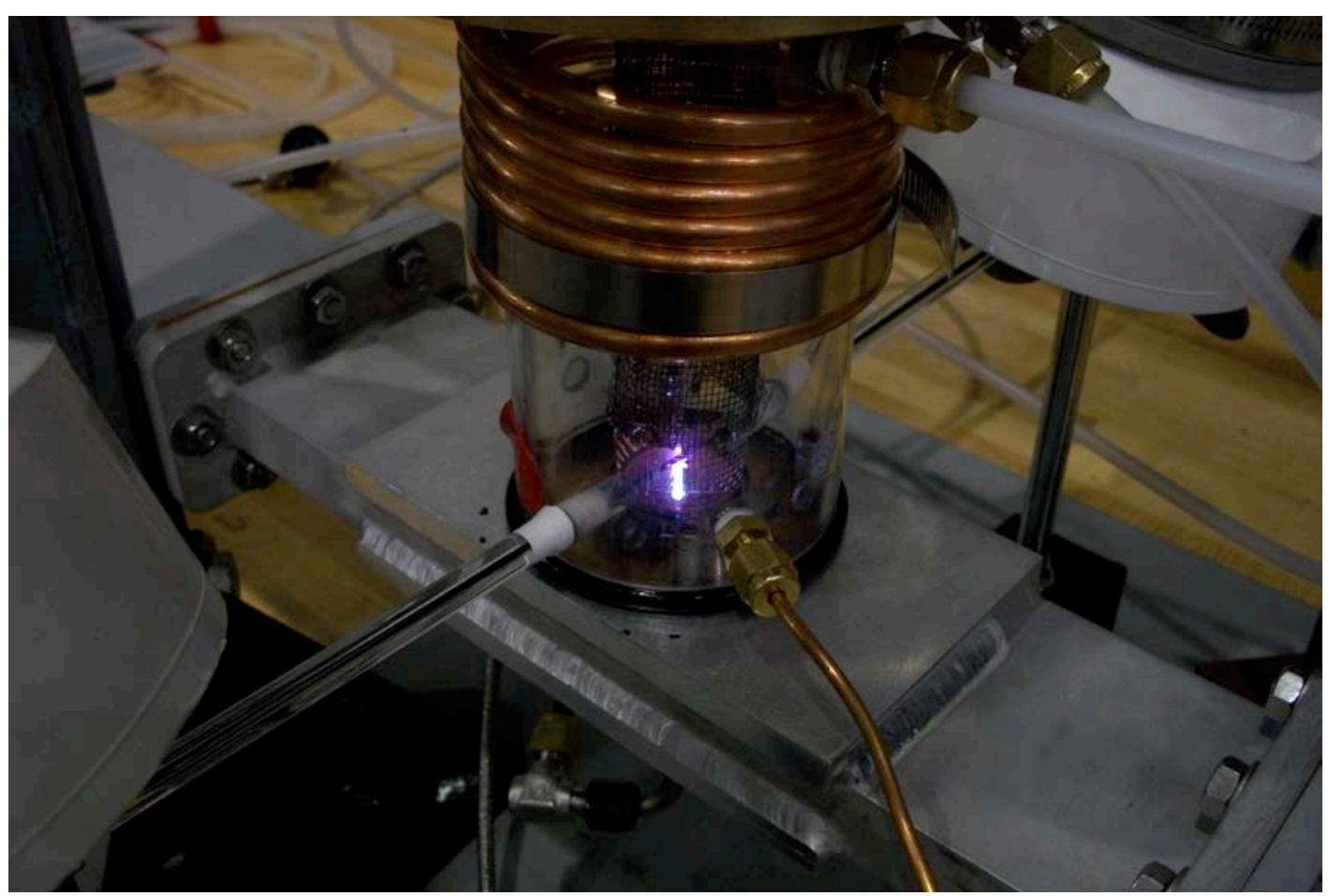

Fig. 29. Atmospheric pressure plasma torch operating with an oxidized tow under inert atmosphere. The copper coil at the upper center is a heat exchanger for the "bell jar" enclosure. 
An initial experiment with oxidized fiber showed fiber temperatures of $\sim 400^{\circ} \mathrm{C}$ at $\sim 70 \mathrm{~W}$ of input power. However, increasing the power led to the destruction of the tow as unfortunately, oxidized fiber placed in the torch was quickly destroyed. Several follow-up experiments were performed after upgrading the seals to further reduce oxygen contamination. At $\sim 80 \mathrm{~W}$, diagnostics indicated the presence of $\mathrm{HCN}$ in the exhaust, so it was concluded that some amount of thermal processing is taking place. However, the temperature of the tow remained below the threshold of the diagnostic, about $400^{\circ} \mathrm{C}$. Increasing the power past $100 \mathrm{~W}$ results in the disintegration of the tow, with no apparent increase in tow temperature before destruction. With no obvious path forward, the decision was made to end efforts to carbonize fiber with a "surfaguide" plasma torch.

Preliminary experiments were also conducted to determine if processing via induction heating could act as a viable pre- or post-heater to the MAP process. The first experiment as shown in Fig.30 used a fully carbonized $12 \mathrm{k}$ fiber whose low resistivity allows for maximum current flow through the fiber.

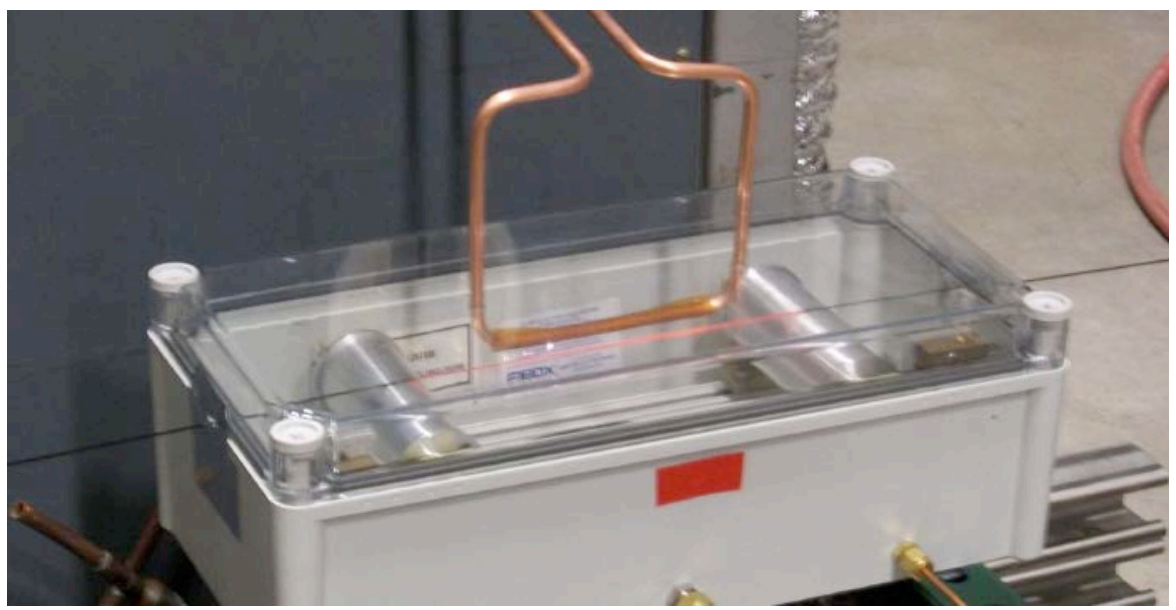

Fig. 30. Heating a carbonized fiber via induction heating.

As shown in Fig. 30, an antenna oriented axially to the fiber can excite enough current to heat the tow to dull red, and by increasing the power to the antenna, the fiber glows incandescent. Though no direct temperature measurement has been done, the temperatures achieved should be hot enough for mid-to-high temperature carbonization. In the preliminary experiments, several issues were encountered. First, some filament breakage was experienced at the interface between the tow and electrode/rollers when the tow was hot. This is probably due to the abrupt temperature gradient of the tow at the electrode. This breakage could be mitigated either by heating the electrode or by directing cooler cover gas at the electrode-tow interface. Second, arcing between the tow and antenna was also observed, some of which is due to broken filaments acting as coronal enhancers. Most of the arcing was eliminated by placing a dielectric material between the antenna and tow and some success was achieved in initial experiments.

After the successful preliminary experiment with fully carbonized fiber, experiments were conducted with less processed fiber. Unfortunately, at the maximum power available with the induction heater, partially processed fiber could not be heated to carbonization temperatures. The resistivity of these feedstocks is simply too high to inductively heat. After this difficulty, the feedstock was switched to tow that had been partially processed with the MAP line. Part of this tow did heat effectively with the induction heater, but a majority of the tow did not. Because of the current level of filament breakage and tensioning issues with MAP-processed fiber, most of the tow in these preliminary experiments 
did not make good electrical contact with the electrode, preventing a closed circuit. Thus, only the part of the tow under tension was heated. The setup was modified in order to mitigate this problem, but significant advantage was not achieved. At the point that this work was concluded, the experiments to date did show some processing capability for induction heating. However, it was determined that the energy consumption and overall footprint requirements would likely be too high for replacing major portions of the carbonization process and at best this approach would only be applicable in final portions of processing already partially carbonized fiber.

The primary alternative processing approach evaluated in this project was based on atmospheric plasma technology developed and demonstrated in collaboration with an ORNL partner for oxidative stabilization of precursor. (Oxidative stabilization is not applicable to the polyolefinic precursors of focus in this work.) In work at partner ReMaxCo on the atmospheric plasma approach, the initial investigation into producing a high intensity plasma discharge with sufficient power density to carbonize oxidized PAN fiber utilizing inductive coupling resulted in the conclusion that 1) generating such a plasma was technically possible, however 2) scaling the resulting optimized apparatus to the industrial scale needed for commercialization would not be economical. Based on this conclusion, the development team switched to the second approach largely employing capacitively coupled plasma. Preliminary analysis indicated that this approach could be scaled to industrially relevant levels in similar fashion to the plasma oxidation technology.

ReMaxCo designed and built necessary hardware to investigate the feasibility of the new fiber carbonization mechanism utilizing near-field capacitively-coupled RF plasma with integrated electromagnetic heating. Significant research into the power electronics portion of this novel processing apparatus was necessary to determine the optimal power signal conditioning and matching to the fiber treatment chamber equipment. Working at this combination of frequency and voltage level has not been done before for this type of application. After extensive experimentation with a wide variety of custom-built power electronics equipment, a solution has been found that provides very good matching and conditioning to the load cell, and which takes advantage of the distributed element (transmission line) domain physics demanded by the operating frequency. This custom piece of equipment as shown in Fig.31, designed and fabricated at ReMaxCo, was built to provide sufficient voltage and power to generate the heating and processing necessary for carbonization of a single $3 \mathrm{k}$ tow of oxidized PAN fiber in a batch process.

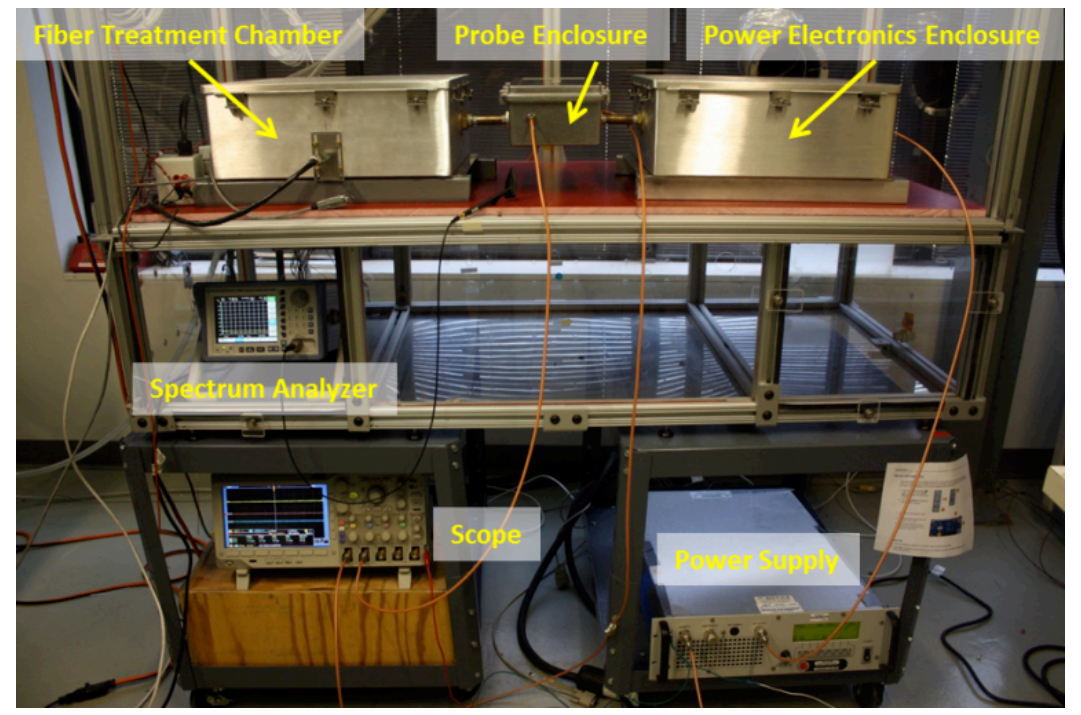

Fig. 31. Atmospheric plasma carbonization experimental setup at ReMaxCo. 
Version 6 of the carbonization system is shown in Fig. 32 below, and can process 4 x 24k oxidized PAN fiber (OPF) tows. The research team was successful at heating the fiber to nearly $500^{\circ} \mathrm{C}$ using this experimental technique. This temperature measurement probably did not capture the maximum temperature actually experienced by the OPF being tested, and as Fig. 33 shows, portions of the fiber were vaporized. This occurred in less than a minute while consuming $50-60 \mathrm{~W}$ of power.

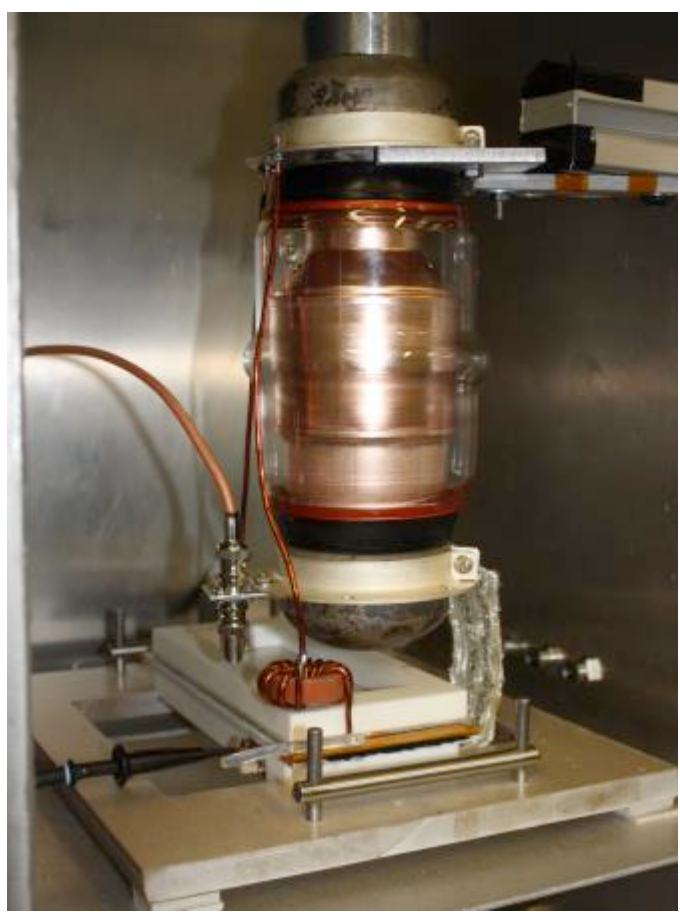

Fig. 32. Version 6 of the benchtop carbonization unit.
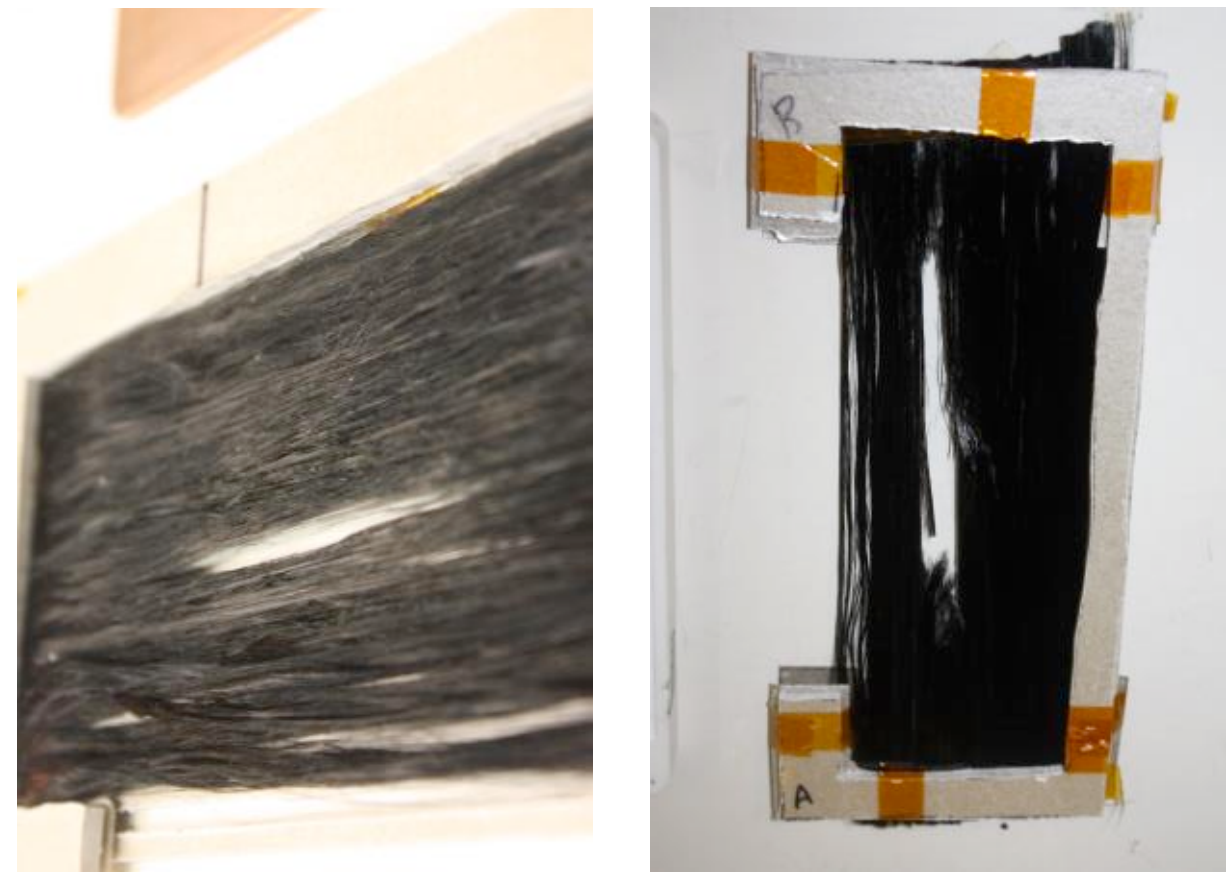

Fig. 33. Samples of partially processed/vaporized OPF. 
It is difficult to accurately measure the density of a very small amount of material, even with liquid density columns. Fortunately, ReMaxCo has been working on a technique utilizing FTIR spectroscopy to measure density of OPF that requires only milligrams of material, and allows for sectioned measuring of OPF density along a tow length (in this case, $7-1 \mathrm{~mm}$ length cuts from a $15 \mathrm{~cm}$ section of processed $24 \mathrm{k}$ OPF tow). Utilizing FTIR also allows for the study of the chemistry of the processed fiber. Results did show an increase of density in certain areas.

This approach is promising. It is flexible, economical, and scalable. It operates at atmospheric pressure. It appears that the temperature requirements for low temperature carbonization can easily be achieved, and high temperature carbonization is possible. Shielding for electro-magnetic interference (EMI) will be required given the frequency range this system operates in, but this is not a major issue. The entire system can be segmented to tune the system to the various stages of processing. The primary deficiency in the system is the generation of hot spots although the research team has proposed three methods of reducing this issue. The plasma component of the system needs to be studied in greater detail to determine exactly its effect on the carbonization process. Once the heating mechanism is optimized, plasma effects can be singled out by proper design-of-experiments methodologies.

Results confirmed that, at the very least, this technique accomplishes "low temperature" carbonization (conventionally in the $500-700^{\circ} \mathrm{C}$ range of processing). The final design (for this project) has been termed the Close-Proximity Electromagnetic-Heating for Carbonization (CPEC) technique. The project team concluded that the new technique is feasible for assisting in low temperature carbonization, but not adequately advanced for implementation in the scope of this project. It is intended to revisit this approach later for longer-term development and demonstration as funding becomes available for next generation technology. 


\section{ACCOMPLISHMENTS AND CONCLUSIONS}

The project team made significant accomplishments in advancing both the development of polyolefinic formulations and fibers for use as carbon fiber precursors as well as the development of advanced carbonization techniques for conversion of stabilized precursors into carbon fiber. In going into this project, ORNL had demonstrated the basics of both approaches at the bench scale but the results at that point were very inconclusive as to capability to scale either approach to commercially viable technologies. At project initiation ORNL had demonstrated the sulfonation approach for stabilizing very small samples of common polyolefinic precursors melt spun under contract to ORNL specifications and then converted into carbon fiber. Although ORNL had advanced the mechanical properties significantly, they were short of automotive minimum targets. All of the spinning, stabilization, and conversion processes had been conducted in small batch-scale processing. Working together, Dow and ORNL evaluated a very wide selection of PO formulations from the vast array of Dow commercial and developmental product lines and selected candidates most appropriate for scaling to commercial-scale production of carbon fiber. Many of these formulations were then spun into fiber at Dow and more fully evaluated in stabilization, carbonization, and mechanical testing by both organizations. The bench-scale batch approach to sulfonation developed by ORNL was converted by the team to continuous processing at Dow that, while still at relatively small-scale, facilitated projection of requirements for the complete process at commercial scale for economic analysis. More importantly the project team developed key understanding of formulation/processing relationships necessary to meet the minimum performance targets and move towards performance between the minimum required for introduction and goals that should make the lower cost forms more attractive in other application areas.

As the team met the minimum mechanical property targets in the final project year as specified by the US automotive industry, Dow was completing its updated evaluation of the economic and technical tradeoffs necessary to make a commercial launch decision. The updated economic projections at Dow indicated that although cost benefits were expected, the projected level of the benefits was likely not sufficient to entice the large capital investment projected for commercial-scale implementation of the baseline sulfonation process utilized for much of this work. Although minimum programmatic property targets were achieved and the precursor development work was on schedule to meet final property milestones, alternative processing routes are desirable to mitigate investment risks. Therefore, the precursor development work was placed on hold pending resolution of Dow assessment of alternatives and plans forward in this area.

ORNL had demonstrated the basics of the microwave assisted plasma process earlier in initial feasibility work funded by VTP. During this feasibility work, the process was primarily demonstrated with a single tow with limited processing of up to three tows simultaneously as a maximum. The process was typically stable for only a few minutes at a time, adequate enough to produce only a few feet of converted carbon fiber for testing. Process durability defined as being able to run continuously stable over at least several hours with repeatable properties from tow-to-tow and along each tow were acknowledged as being short falls that needed to be overcome on the pathway to commercialization along with a proven ability to process more than a single tow in each module. This project tackled these issues and demonstrated that these goals can be met.

The goal of the advanced conversion portion of this project is to demonstrate the technology necessary to move to the next stage of scaling to commercialization. Based on feedback from carbon fiber manufacturing insiders and the end user community, a key target was to demonstrate technology readiness levels commensurate with having the information necessary to move forward to preproduction scale consistent with production capabilities recently introduced at the ORNL Carbon 
Fiber Technology Facility in hand at the conclusion of the project. This capability is defined by 24 tows of $24,000(24 \mathrm{~K})$ tows running at about $60 \mathrm{in} / \mathrm{min}$ or similar combinations. The project team with sponsor concurrence defined the deliverable evidence of meeting this goal as production of a technical specification for equipment to go into CFTF for producing carbon fiber for evaluation purposes (both equipment as well as carbon fiber product) at this scale.

Going into the project, one of the key issues inhibiting scaling was lack of capability to distribute the energy as needed in the progression of fiber in going from a non-conductive oxidized (or stabilized) precursor to completed carbonized and relatively conductive carbon fiber. Related to this difficulty was the need to evenly distribute the energy across tows being processed concurrently. Early in this project the team established the capability to measure dielectric properties of fibers at various stages of conversion as a function of the temperature environment. Dielectric properties influence coupling in an electromagnetic field, which allows basic modeling of the process. Although the dynamic nature of this process in combination with the complexity of manipulating the plasma and microwave heating mechanisms make this a very complicated problem suitable for a large research project in itself, the team was able to develop and utilize models to assist with hardware development. Using the modeling as well as materials and processing data experiments, the team essentially optimized the original MAP process for accomplishing both low and high temperature carbonization processes (in conventional thermal processing these tow processes are typically performed in discrete steps in totally separate furnaces) for up to five tows up to about $48 \mathrm{in} / \mathrm{min}$.

Although the team was continuing to make progress with the baseline MAP approach, it was recognized the gains were becoming much more incremental in nature and that alternatives might make this process more attractive. Early on the team acknowledged that the low temperature carbonization with much higher effluent generation would be the greatest challenge for the MAP process operating at the low pressures required to generate and maintain a stable plasma. Several approaches were evaluated and a team led by long-term partner ReMaxCo demonstrated basic feasibility for what is described as Close-Proximity Electromagnetic-Heating for Carbonization (CPEC) that works exclusively in the low temperature portion of the carbonization. This process was identified as having great potential, but was judged to be too immature for concentration in the final demonstration phase of this project. With this verdict, work on alternative low temperature carbonization process alternatives was deferred for later consideration.

During this time frame an alternative technique for dividing and controlling sequential energy deposition in multiple chambers was proposed and evaluated. Initial work was even more promising than expected and this technique was expanded to demonstrate its effectiveness over three-chamber deposition with a single cylindrical processing reactor. With this configuration the team was able to produce converted carbon fiber at $60 \mathrm{in} / \mathrm{min}$.

Other issues of importance to scaling are overall energy efficiency and effective effluent handling. At each point of scale-up in going to more tows and higher speeds the generation of effluent has increased significantly. At each step in the progression of this work, new approaches to effluent handling were developed and implemented. While the team will not claim this issue has been resolved, it is felt that significant improvements were implemented at several points to get to the next step and that standard industry approaches in production may be capable of being modified for the advanced processes, with proper attention to pumping issues. There is no reason to believe that this process generates any more effluent that the standard thermal processing and the plasma may possibly have a somewhat mitigating effect. However, effluent handling will be a key issue to tackle in the scaling to the next level of production.

During the course of the project the team did demonstrate capability of the process to be operated for 
extended periods of time. Although it is believed that a key inherent advantage of the MAP (and the CPEC) process is the capability to stop and start rapidly, greatest efficiencies will come from continuous operations. Two-hour and eight-hour continuous operating milestones were achieved with little difficulty during this project and the team saw no signs of instabilities or that operational limits were being approached.

The project team believes the advanced carbonization technology is capable of meeting the previously outlined targets for scaling to the next level. The deliverable project specifications for scaling the carbonization system to the 25-ton-per-year CFTF production level have been drafted and can be shared with interested parties. However, due to the specification having both export controlled processing information and background for potential patent applications, this information cannot be released publicly.

\subsection{INTELLECTUAL PROPERTY}

ORNL intellectual property contributions were brought into performance of the CRADA as background intellectual property. Amit Naskar and Felix Paulauskas filed an invention disclosure with ORNL, "Microwave Processing of Functionalized Polyolefin Fibers" in September of 2010. Based on the initial feasibility studies on the atmospheric plasma investigation, invention disclosures entitled "Atmospheric Plasma Carbonization of Pre-Stabilized/Oxidized Precursors" and "Multicavity Microwave Processing Using a Single Microwave Source" have been submitted for patent filing considerations.

As a result of the research conducted under this project, Dow has filed patent applications for the following subject inventions (Fig. 34).

\begin{tabular}{|l|c|c|}
\hline \multicolumn{1}{|c|}{ Title } & US Serial Number & Date \\
\hline $\begin{array}{l}\text { Process for Preparing Carbon Fibers Using Sulfur } \\
\text { Trioxide in a Halogenated Solvent }\end{array}$ & $61 / 670802$ & $7 / 12 / 2012$ \\
\hline $\begin{array}{l}\text { Processes for Preparing Carbon Fibers Using } \\
\text { Gaseous Sulfur Trioxide }\end{array}$ & $61 / 670810$ & $7 / 12 / 2012$ \\
\hline $\begin{array}{l}\text { Two-Step Sulfonation Process for the Conversion of } \\
\text { Polymer Fibers to Carbon Fibers }\end{array}$ & $61 / 670821$ & $7 / 12 / 2012$ \\
\hline
\end{tabular}

Fig. 34. Dow patents applications resulting from this project.

\subsection{PUBLICATIONS AND PRESENTATIONS}

R. Norris, et al., "Development and Commercialization of Alternative Carbon Precursors and Conversion Technologies" ORNL CRADA Report, NFE-10-02991, October 2013.

\subsection{CONLCUSIONS}

ORNL and Dow combined in this project to make substantial inroads in the development of new carbon fiber precursors and conversion technology and towards establishing pathways to commercialization of these interrelated technologies. The technology to produce alternative polymer fiber precursor formulations as well as scaled energy-efficient advanced conversion technology will facilitate commercialization of carbon fibers that are technically and economically viable in industrial markets such as transportation, wind energy, gas storage, infrastructure and oil drilling applications. 
Although there have been efforts in the past to produce a low cost carbon fiber, current carbon fiber production techniques are currently derived from historical needs of military aircraft and high-end aerospace components which are not conducive of meeting cost targets of commercial energy-related missions. This approach is different in that the researchers were free to adjust both manufacturing process and precursor chemistry to meet the different physical specifications at a lower cost.

During the course of the project, the precursor development team moved from utilization of commercially available sources of polyolefinic materials identified earlier by ORNL to specific formulations chosen by the team as most likely to meet technical and economic goals of the project from the vast reservoir of Dow sources. The team developed appropriate screening criteria that was implemented and adjusted as appropriate in making materials selection. The sulfonation process previously utilized in batch form by ORNL was implemented in continuous form by the project team and property versus property relationships were established. With enhanced knowledge and experience of the team, the best formulation alternatives were identified and Dow provided fibers custom spun and stabilized through sulfonation for conversion and testing by ORNL and Dow. Properties were advanced to levels approaching program targets when Dow economic analyses indicated that the sulfonation approach made large-scale economic advantages marginal versus assumed risks. At this point, the polyolefin development work was suspended while Dow assessed possibilities for alternative stabilization techniques.

Work on the advanced conversion process has continued to advance the baseline technology to the point where the team believes scaling to the pre-production level is the next step. Various alternative approaches to atmospheric plasma processing using plasma torch approaches as well as inductively and capacitively coupled plasmas have been considered. Although encouraging results were achieved with the capacitively-coupled approach, this technique was considered not sufficiently mature for scaling at this time, but with further development, may be reconsidered in the future. A non-plasma inductive technique for preheating and/or post-processing was evaluated as well, but did not provide significant advantages for further exploitation at this time. At the time of this report, the pilot-scale unit constructed with a single low pressure processing chamber utilizing multiple microwave cavities integrated for enhanced energy deposition control has demonstrated especially encouraging results giving the team confidence that speeds comparable with the CFTF lines are achievable and that implementing modules in parallel fed from a single microwave generator should be adequate for matching the volumetric production level targets. Earlier work focusing on running for extended periods with the original energy deposition approach appears to be transferable to the better deposition system demonstrated at project conclusion.

Utilizing advances described in this report, low pressure sealing techniques have been improved and external cooling and effluent trapping approaches demonstrated to enhance process durability. In addition to better understanding of energy deposition itself, the modeling work has assisted in better understanding of how tow placement impacts process uniformity. Minimum property level targets have been consistently achieved at each stage of the hardware advancements described and property uniformity appears within reach when adequately uniform precursor is available. A baseline energy balance has been developed to assist in evaluation of economics of the final demonstration system with semi-optimized processing conditions. The team has demonstrated and discussed the advanced conversion approach with potential commercialization partners and specifications have been developed for pre-production scale equipment. Although these discussions are ongoing at the time of this report, initial response indicates interest in collaboration from both the carbon fiber manufacturing community as well as from suppliers of conventional carbon fiber manufacturing equipment.

During the course of the project, ORNL completed and commissioned the 25-tonne-per-year CFTF 
with DOE funding outside this work. This capability will enhance demonstration of carbon fiber technologies at near-production scale in order to facilitate commercialization of these technologies. It is intended for this capability to be utilized in paving the pathway forward for introduction of low cost precursors such as polyolefins and as a location for demonstration of the next-scale for the advanced conversion process. 


\section{COMMERCIALIZATION}

Carbon fiber manufacturing has been identified as a technology of strategic importance to the United States. In addition to aerospace and defense importance, it is generally conceded that carbon fiber composites have potential to make significant impact in energy missions such as vehicle lightweighting, enabling longer and more efficient wind turbine blades, enhanced structural capacity for offshore oil and gas exploration and production structures, etc. For example, studies have shown that for $10 \%$ structural weight reduction in automotive applications would typically provide a $5-7 \%$ improvement in gas mileage. However, cost of the carbon fiber itself has been a limiting factor for applications in these areas. In a cost analysis study conducted by Kline and Company, it has been shown that the carbon fiber cost to manufacture can be reduced toward $\$ 5-8 / \mathrm{lb}$ if low-cost precursors and advanced technology such as plasma oxidation and Microwave Assisted Plasma (MAP) carbonization routes are adopted. ${ }^{1}$ The study also revealed that manufacturing process costs make up more than $50 \%$ of the total cost of commercially available carbon fibers. This is primarily due to the low carbon yield of the precursors $(<50 \%$ of the mass of the precursor becomes carbon fiber). Prior work conducted by Hexcel researchers (2004) indicated 78\% carbon yield from polyolefin precursor; therefore, about $50 \%$ increase in production rate could be expected if polyolefin-based precursors are considered for low-cost carbon fiber (LCCF) production ${ }^{2}$. Work in advanced conversion has demonstrated a $2 \mathrm{X}$ improvement in residence time that would translate into smaller equipment per unit of production capacity with inherently less energy required in processing. In summary, there is a very high potential that LCCF can be economically produced from polyolefin precursors with advanced conversion techniques at a substantial cost reduction over other alternative precursor materials and utilizing conventional manufacturing processes.

Utilizing DOE funding outside of this project, ORNL completed and commissioned a 25-tonne-peryear Carbon Fiber Technology Facility (CFTF) in 2013. This capability was established to enhance demonstration of carbon fiber technologies at near-production scale in order to facilitate commercialization of LCCF technologies. Goals for this project were to target utilization of CFTF capabilities in scaling to pre-production scale demonstration of both alternative precursors and advanced conversion technologies in the next phase of moving each of these approaches to commercialization. As such, a team led by Dow has sought and received funding to begin scaling conversion of alternative precursors demonstrated in this project to pre-production scale at CFTF and a deliverable of the advanced conversion effort is the specification for equipment that can be implemented at CFTF based on the new technology in comparable size ranges.

In addition to the higher carbon yield, polyolefinic precursors offer significant advantage in that they are melt-spun versus requirement for PAN-based precursors to be solution spun. Melt spinning offers significant advantages in terms of overall throughput, much more favorable environmental conditions without need for solvent recovery and handling, smaller footprint without needs for solvent recovery space, and reduced energy consumption primarily due to the higher throughput and reduced solvent processing needs. However, as identified during the project, the stabilization process for polyolefinic-based precursors is significantly different than the oxidative stabilization process employed for PAN precursors. It has been postulated prior to this project and then confirmed during this project that the sulfonation can be completed in residence times and process speeds comparable

1 Cost modeling analysis of carbon fiber manufacturing by Kline and Company (for Automotive Composite Consortium), Annual report 2007.

2 M. G. Abdallah, B. Hansen, G. Jacobsen, Low Cost Carbon Fiber (LCCF) Development Program, Phase-1 final submitted to U.S. DOE/OTT Automotive Lightweighting Materials Division (Contract Sponsor) via the Oak Ridge National Laboratory (Technical Administrator for DOE/OTT) (2004). 
to those required for PAN-based precursors. However, as Dow carefully examined the economics required for implanting commercial-scale stabilization, it was concluded that although cost benefits were expected overall in the production of carbon fibers from polyolefinic precursors, the projected level of the benefits was likely not sufficient to entice the large capital investment projected for commercial-scale implementation of the baseline sulfonation process. Although minimum programmatic property targets were achieved and the precursor development work was on schedule to meet final property milestones, alternative processing routes are desirable to mitigate investment risks. During the course of this work, ORNL had identified and was evaluating a gas-phase alternative to the baseline sulfonation process and Dow has identified other approaches that might be applicable to this portion of the conversion process. To advance further towards commercialization, additional work will be required to assess and/or improve the baseline sulfonation process for commercial scale production or alternative process approaches must be demonstrated as technically and economically attractive.

During the course of the project ORNL had had many discussions with existing carbon fiber manufacturers and carbon fiber manufacturing equipment suppliers as well as with others interested in joining this industry as to the basic approach ORNL has been developing for advanced conversion. However, as the nature of the alternative technology was advancing significantly during the project period and the technology itself has both intellectual property and export controlled ramifications, the initial discussions were held at high levels only describing goals and accomplishments in terms of production rates and fiber properties without providing details of hardware design and processing parameters. These details are critical in evaluating potential for understanding and projecting risks and rewards for large-scale implementation. As the team established and validated details for the new baseline equipment demonstrated in the final project activities, formal efforts were initiated to recontact organizations previously identified as potential stakeholders in going to the next step towards commercialization. An overview package has been prepared describing the new technology and some of the processing details for discussion with interested parties. Although it has not been shared with others at the time of the report, the specification package for the equipment at CFTF scale can also be utilized in discussions on plans for going forward.

A number of existing carbon fiber manufacturers and carbon fiber manufacturing equipment suppliers have been contacted to provide feedback on what has been accomplished and to determine interest in more formal discussions concerning supporting or partnering in scaling up this process. To formally discuss moving forward, organizations having interest were asked to sign a non-disclosure agreement. At the time of this report, three organizations have signed the agreement and these discussions are ongoing. One of these organizations has already visited ORNL and viewed the system in operation leading to initiation of a materials exchange to compare properties of conventionally processed fiber versus those of fiber from the advanced conversion process. Discussions with this company and others that have signed the agreement also involve how to get comparable numbers on energy utilization and effluent generation and these companies can provide expertise in addressing related needs in going forward should they continue to stay engaged.

While it is not yet known if companies currently in discussions concerning this technology will be willing to provide significant financial or in-kind support in scaling to the next level, it is clear that there is interest in further evaluation of the capabilities of this technology at production levels exceeding what is currently available from the existing system. Although it is clear that residence time is a critical factor in evaluating this technology and this appears to be a key advantage for this technology, feedback from one company indicates they would be most interested in exploring much higher line speeds than those comparable to CFTF production rates, albeit the 25-tonne-per-year ultimate level for pre-production demonstration might still be appropriate. In going to significantly higher line speeds the team believes the alternative low temperature carbonization process identified 
earlier should be reconsidered for integration with the new baseline results of this project. As this report is being completed, additional potential partners are being approached to assess interest and a more detailed proposal and plan for scaling the technology and demonstrating capabilities at the next higher production rate level is being prepared for discussion among sponsors and potential partners. 


\section{RECOMMENDATIONS}

ORNL believes there is merit in advancing both the polyolefinic precursor development and the advanced carbonization technologies. The cost of the polyolefin base polymer, cost of the melt spinning and carbonization processes, and the resulting high carbon yield all tilt in favor of PO-based materials being attractive as carbon fiber precursors if an acceptable stabilization approach can be found. Due to questions concerning the economical attractiveness of scaling the sulfonation process that was focus of this effort, follow-on efforts should focus on alternative methods such as a gasphase process or other means for rapidly stabilizing the PO material with less environmentally challenging materials than utilized in the baseline sulfonation process. Alternative approaches might be initially explored through smaller and more basic projects such as SBIRs, incubator funding, or seed money efforts. Much of materials selection and carbonization process development accomplished in this project should be applicable to follow-on work should a potentially attractive stabilization process be identified.

The team strongly recommends moving the advanced carbonization process along the next step(s) towards commercialization. Prior to actually designing and building modules for the higher production levels consistent with CFTF, the team should explore approaches for running at significantly higher line speeds. Preliminary industry feedback is that targeting very high line speeds might be more attractive to the industry than meeting the previously identified pre-production rates at current CFTF line speeds. Although shorter actual residence times for carbonization have been shown in this work, 1) approaches for running at higher line speed will likely require adding cavities in a series versus 2) scaling the new baseline to higher production levels while matching CFTF line speed requires adding MAP modules in parallel. It is also likely that the alternative CPEC low temperature carbonization process will facilitate operations at higher speeds since operating at atmospheric pressure levels makes effluent removal somewhat simpler. Because this technology is much less mature than the new baseline process, scaling of the low temperature carbonization process will need to quickly catch up and surpass current processing levels for the baseline to meet the ultimate scaling targets. The team recommends working on issues associated with adding additional cavities in series for the high temperature carbonization process utilizing materials that have already been processed through a representative low temperature carbonization process in parallel with verifying that the CPEC process can be scaled to match the current baseline. The baseline lowpressure approach will be required for the high temperature carbonization process regardless of the success for the atmospheric pressure CPEC approach in low temperature carbonization. With these results in hand the complete carbonization process can be more effectively scaled to meet the preproduction level. The team believes continuing to strengthen teaming relationships with carbon fiber producers and carbon fiber production equipment manufacturers will enhance probability both of technical success in scaling and longer-term success in moving this technology into commercialization. 